Provided for non-commercial research and education use. Not for reproduction, distribution or commercial use.

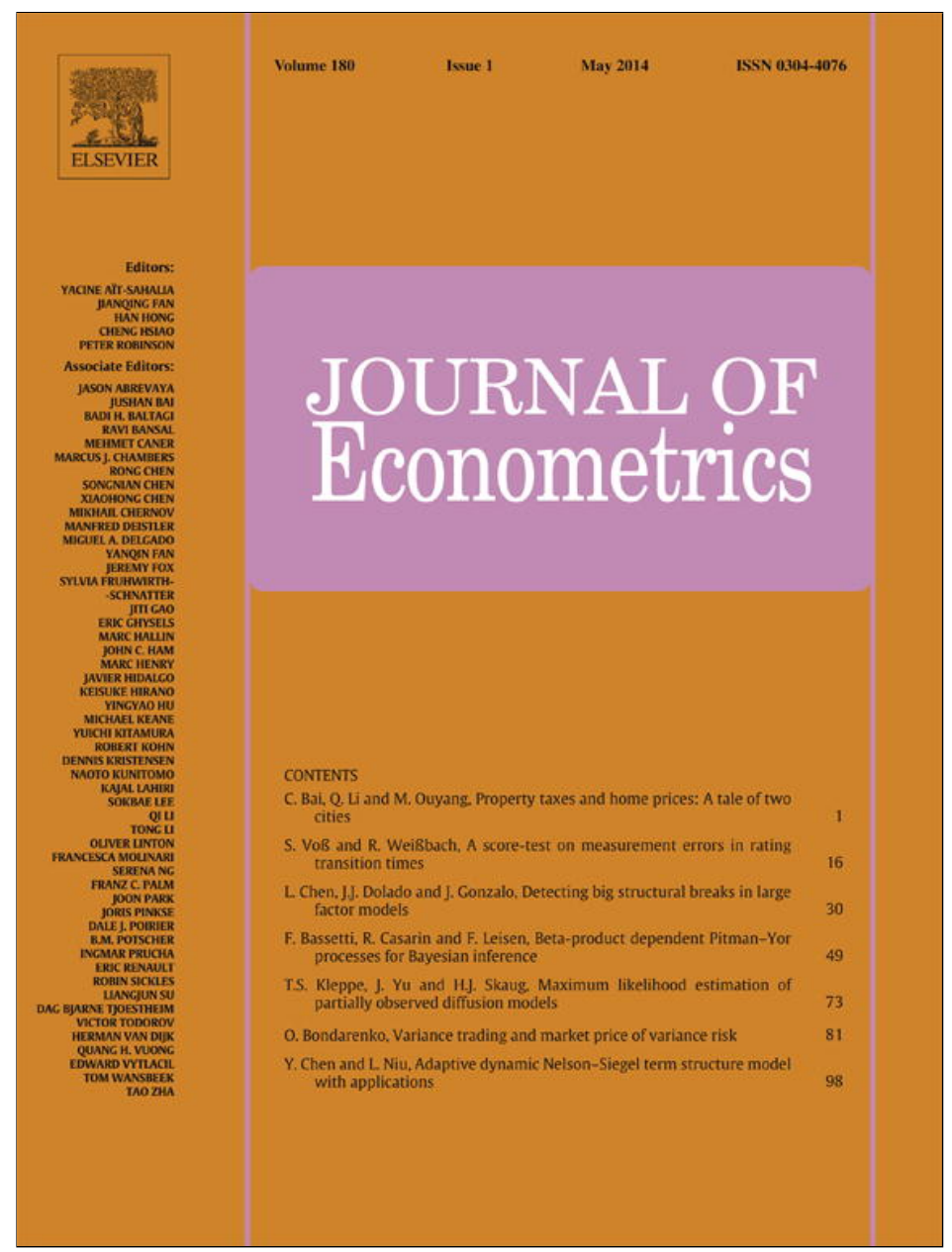

This article appeared in a journal published by Elsevier. The attached copy is furnished to the author for internal non-commercial research and education use, including for instruction at the authors institution and sharing with colleagues.

Other uses, including reproduction and distribution, or selling or licensing copies, or posting to personal, institutional or third party websites are prohibited.

In most cases authors are permitted to post their version of the article (e.g. in Word or Tex form) to their personal website or institutional repository. Authors requiring further information regarding Elsevier's archiving and manuscript policies are encouraged to visit:

http://www.elsevier.com/authorsrights 


\title{
Detecting big structural breaks in large factor models $\mathbf{s}^{\text {* }}$
}

\author{
Liang Chen ${ }^{\text {a }}$ Juan J. Dolado ${ }^{\text {b,c }}$, Jesús Gonzalo ${ }^{\text {b,* }}$ \\ ${ }^{a}$ Department of Economics, Nuffield College and Institute of New Economic Thinking, University of Oxford, United Kingdom \\ ${ }^{\mathrm{b}}$ Department of Economics, Universidad Carlos III de Madrid, Spain \\ ${ }^{\mathrm{c}}$ European University Institute, Italy
}

\section{A R T I C L E I N F O}

\section{Article history:}

Received 9 May 2012

Received in revised form

3 October 2013

Accepted 21 January 2014

Available online 31 January 2014

\section{JEL classification:}

C12

C33

Keywords:

Structural break

Large factor model

Factor loadings

Principal components

\begin{abstract}
A B S T R A C T
Time invariance of factor loadings is a standard assumption in the analysis of large factor models. Yet, this assumption may be restrictive unless parameter shifts are mild (i.e., local to zero). In this paper we develop a new testing procedure to detect big breaks in these loadings at either known or unknown dates. It relies upon testing for parameter breaks in a regression of one of the factors estimated by Principal Components analysis on the remaining estimated factors, where the number of factors is chosen according to Bai and Ng's (2002) information criteria. The test fares well in terms of power relative to other recently proposed tests on this issue, and can be easily implemented to avoid forecasting failures in standard factor-augmented (FAR, FAVAR) models where the number of factors is a priori imposed on the basis of theoretical considerations.
\end{abstract}

(c) 2014 Elsevier B.V. All rights reserved.

\section{Introduction}

Despite being well acknowledged that some parameters in economic relationships can be subject to important structural breaks (e.g., those related to technological change, globalization or strong policy reforms), a standard practice in the estimation of large factor models (FM, hereafter) has been to assume timeinvariant factor loadings. Possibly, one of the main reasons for this benign neglect of breaks stems from the important results obtained by Stock and Watson $(2002,2009)$ regarding the consistency of the estimated factors by principal components analysis (PCA hereafter) when the loadings are subject to small (i.e., local-tozero) instabilities. These authors conclude that the failure of factorbased forecasts is mainly due to the instability of the forecast

\footnotetext{
We are grateful to a co-editor, two anonymous referees, Dante Amengual, Manuel Arellano, Stephane Bonhomme and Soren Johansen for insightful comments, and to Atsushi Inoue for helpful discussions on his related but independent research. We also thank seminar participants at Aarhus, CEMFI, Conference in Honor of Sir David F. Hendry (St. Andrews), ESEM 2011 (Oslo), and Workshops on High-Dimensional Econometric Modelling (Cass Business School) and Time Series Econometrics (Zaragoza) for suggestions. Financial support from the Spanish Ministerio de Economía y Competitividad (grant ECO2010-19357), Comunidad de Madrid (grant Excelecon), Bank of Spain (grant ER program), Open Society Foundations and the Oxford Martin School, is gratefully acknowledged.

* Corresponding author. Tel.: +34 916249853.

E-mail addresses: liang.chen@economics.ox.ac.uk (L. Chen), dolado@eco.uc3m.es (J.J. Dolado), jesus.gonzalo@uc3m.es (J. Gonzalo).
}

http://dx.doi.org/10.1016/j.jeconom.2014.01.006

0304-4076/C 2014 Elsevier B.V. All rights reserved. function, rather than of the different components of the FM. As a result, their advice is to use full-sample factor estimates and subsample forecasting equations to improve forecasts.

However, the main emphasis placed on local-to-zero breaks has been subsequently questioned. For example, by means of a Monte Carlo study, Banerjee et al. (2008) conclude that, in contrast to Stock and Watson's diagnosis, the instability of factor loadings when big (i.e., not local-to-zero) breaks occur is the most likely reason behind the worsening factor-based forecasts, particularly in small samples. Likewise, when discussing Stock and Watson's research on this topic, Giannone (2007) argues that “... to understand structural changes we should devote more effort in modelling the variables characterized by more severe instabilities...". In this paper, we pursue this goal by providing a precise characterization of the different conditions under which big and small breaks in the factor loadings may occur, as well as develop a simple test to distinguish between them. We conclude that, in contrast to small breaks, big breaks should not be ignored since they may lead to misleading results in standard econometric practices using FM and in the potential interpretation of the factors themselves.

A forerunner of our paper is Breitung and Eickmeier (2011, $\mathrm{BE}$ henceforth) who were the first to propose a proper testing procedure to detect big breaks in the factor loadings. Their test relies on the idea that, under the null of no structural break (plus some additional assumptions), the estimation error of the factors can be ignored and thus the estimated factors can be treated as the true ones. Consequently, a Chow-type test can be implemented 
by regressing each variable in the data set on both the estimated factors using the whole sample and their truncated versions from the date of the break onwards. Focusing on the joint statistical significance of the estimated coefficients on the truncated factors, their test compares the empirical rejection frequency among the individual regressions to a nominal size of $5 \%$ under the null. In our view, this approach suffers from two limitations: (i) the overall limiting distribution of their test remains unknown when testing for the equality of all the elements of the loading matrix in both subsamples ${ }^{1}$; and (ii) it lacks non-trivial power when the number of factors is chosen according to some consistent estimator of $r$. This last problem can be serious. For example, as explained further below, a FM with $r$ original factors where the loadings of one of them exhibit a big structural break at the same date admits a standard factor representation with $r+1$ factors without a break. Hence, if the number of factors were to be chosen as $r+1$, instead of $r$, their testing approach would fail to detect any break at all when in fact there is one.

Our contribution here is to propose a simple testing procedure to detect big breaks in FMs stemming from a single source which does not suffer from the previous shortcomings. We focus on breaks in the loadings though we also provide a procedure to detect whether the breaks originate from the loadings or from factors themselves. In particular, we first derive some asymptotic results finding that, in contrast to small breaks, the number of factors is overestimated under big breaks, a result which was also used by $\mathrm{BE}$ (2011). We argue that neglecting these breaks can have serious consequences on the forecasting performance of some popular regression-based models using factors, where their number is a priori imposed. Likewise, under big breaks, it may be difficult to provide a structural interpretation of the estimated factors when they are chosen according to some consistent information criteria (see Bai and Ng, 2006b; Chen, 2012). Our proposal relies upon a very simple regression-based testing procedure. As sketched earlier, the insight is that a FM with big breaks in the loadings can be re-parameterized as another FM with constant loadings but a larger set of factors, where the number and the space spanned by the latter can be consistently estimated by PCA under fairly standard assumptions. Hence, rather than directly testing for whether all the elements of the loadings matrix are stable, which will suffer from an infinite-dimensionality problem as the number of variables in the panel data set grows, one can test if the relationships among the larger finite-dimensional set of estimated factors are stable.

Specifically, our procedure consists of two steps. First, the number of factors for the whole sample period is chosen as $\bar{r}$ according to Bai and Ng's (2002, BN henceforth) information criteria, and then $\bar{r}$ factors are estimated by PCA. Next, one of the estimated factors (e.g., the first one) is regressed on the remaining $\bar{r}-1$ factors, to next apply the standard Chow Test or the Sup-type Test of Andrews (1993) to this regression, depending on whether the date of the break is treated as known or unknown. If the null of no breaks is rejected in the second-step regression, we conclude that there are big breaks and, otherwise, that either no breaks exist at all or that only small breaks occur. Further, on the basis of the rank properties of the covariance matrix of the estimated factors in different subsamples, we also provide some guidance on how to distinguish between breaks stemming either from the loadings or from the data generating process (DGP hereafter) of the factors. This difference is important since the latter may lead to reject the

\footnotetext{
1 With the notation used below in (1)-(2), the limiting distribution of the rejection frequencies for the joint hypothesis $A=B$ is not known, although the individual tests for the hypothesis $\alpha_{i}=\beta_{i}$ have known limiting distributions.
}

null of constant loadings when it is true, implying a misleading interpretation of the source of the break.

After completing the first draft of this paper, we became aware of a closely related paper by Han and Inoue (2012, HI hereafter) who, in an independent research, adopt a similar approach to ours in testing for big breaks in FM. The two approaches, however, differ in some relevant respects. In effect, rather than using a simple regression-based approach to avoid the infinite-dimensionality problem as we do here, $\mathrm{HI}$ (2012) test directly for differences before and after the break in all the elements of the covariance matrix of the estimated factors. We will argue below that, despite the fact that the HI tests use more information than ours, both tests generally exhibit similar power. Indeed, our regression-based test based on the Wald principle, which behaves much better in general than the Lagrange multiplier (LM hereafter) tests for detecting structural breaks, is even more powerful than the corresponding HI's test for small sample sizes, such as $N=T=50$. One additional advantage of our simple linear-regression setup is that it amenable to use many other existing methods for testing breaks, including multiple ones (see, e.g. Perron, 2006, for an extensive review of these tests).

The rest of the paper is organized as follows. In Section 2, we present the basic notation, assumptions and the definitions of small and big breaks. In Section 3, we analyze the consequences of big breaks on the choice of the number of factors and their estimation, as well as their effects on standard econometric practices with factor-augmented regressions. In Section 4, we first derive the asymptotic distributions of our tests and next discuss, when a big break is detected, how to identify its sources. Section 5 deals with the finite sample performance of our test relative to the competing tests using Monte-Carlo simulations. Section 6 provides an empirical application. Finally, Section 7 concludes. An appendix contains detailed proofs of the main results.

\section{Notation and preliminaries}

We consider FM that can be rewritten in the static canonical form:

$X_{t}=A F_{t}+e_{t}$

where $X_{t}$ is the $N \times 1$ vector of observed variables, $A=$ $\left(\alpha_{1}, \ldots, \alpha_{N}\right)^{\prime}$ is the $N \times r$ matrix of factor loadings, $r$ is the number of common factors which is finite, $F_{t}=\left(F_{1 t}, \ldots, F_{r t}\right)^{\prime}$ is the $r \times 1$ vector of common factors, and $e_{t}$ is the $N \times 1$ vector of idiosyncratic errors. In the case of dynamic FMs, all the common factors $f_{t}$ and their lags are stacked into $F_{t}$. Thus, a dynamic FM with $r$ dynamic factors and $p$ lags of these factors can be written as a static FM with $r \times(p+1)$ static factors. Further, given the assumptions we make about $e_{t}$, the case analyzed by $\mathrm{BE}$ (2011) where the $e_{i t}$ disturbances are generated by individual specific autoregressive (AR hereafter) processes is also considered. ${ }^{2}$

We assume that there is a single structural break in the factor loadings of all factors at the same date $\tau$ :

$X_{t}=A F_{t}+e_{t} \quad t=1,2, \ldots, \tau$,

$X_{t}=B F_{t}+e_{t} \quad t=\tau+1, \ldots, T$

where $B=\left(\beta_{1}, \ldots, \beta_{N}\right)^{\prime}$ is the new factor loadings after the break. By defining the matrix $C=B-A$, which captures the size of the

\footnotetext{
2 Notice, however, that our setup excludes the generalized dynamic FM considered by Forni and Lippi (2001), where the polynomial distributed lag possibly tends to infinity.
} 
breaks, the FM in (1) and (2) can be rewritten as:

$X_{t}=A F_{t}+C G_{t}+e_{t}$

where $G_{t}=0$ for $t=1, \ldots, \tau$, and $G_{t}=F_{t}$ for $t=\tau+1, \ldots, T$.

As argued by Stock and Watson $(2002,2009)$, the effects of some mild (local to zero) instability in the factor loadings can be averaged out, so that estimation and inference based on PCA remain valid. We generalize their analysis by allowing for two types of break sizes: small and big. In contrast to the former, we will show that the latter cannot be neglected. To distinguish between them, it is convenient to partition the $C$ matrix as follows:

$\mathrm{C}=[\Lambda \mathrm{H}]$

where $\Lambda$ and $H$ are $N \times k_{1}$ and $N \times k_{2}$ matrices that correspond to the big and the small breaks, and $k_{1}+k_{2}=r$. Accordingly, we can also partition the $G_{t}$ matrix into $G_{t}^{1}$ and $G_{t}^{2}$, such that (3) becomes:

$X_{t}=A F_{t}+\Lambda G_{t}^{1}+H G_{t}^{2}+e_{t}$

where $\Lambda=\left(\lambda_{1}, \ldots, \lambda_{N}\right)^{\prime}$ and $H=\left(\eta_{1}, \ldots, \eta_{N}\right)^{\prime}$.

Throughout the paper, $\operatorname{tr}(\Sigma)$ and $\|\Sigma\|=\sqrt{\operatorname{tr}\left(\Sigma^{\prime} \Sigma\right)}$ will denote the trace and the norm of a matrix $\Sigma$, respectively. For a finite dimensional vector $v$, we write $v=O_{p}(1)\left(v=o_{p}(1)\right)$ when $\|v\|=O_{p}(1)\left(\|v\|=o_{p}(1)\right) .[T \pi]$ denotes the integer part of $T \times \pi$ for $\pi \in[0,1]$. Once the basic notation has been established, we next provide precise definitions of the two types of breaks.

Assumption 1. Breaks: (a) $\left\|\lambda_{i}\right\| \leq \bar{\lambda}<\infty$ for all $i . N^{-1} \Lambda^{\prime} \Lambda \rightarrow$ $\Sigma_{\Lambda}$ as $N \rightarrow \infty$ for some positive definite matrix $\Sigma_{\Lambda}$. (b) $\eta_{i}=$ $(N T)^{-1 / 2} \kappa_{i}$ and $\left\|\kappa_{i}\right\| \leq \bar{\kappa}<\infty$ for all $i$.

The matrices $\Lambda$ and $H$ are assumed to contain non-random elements. Assumption 1(a) yields the definition of a big break. It also includes the case where $\lambda_{i}=0$ (no break) for a fixed proportion of variables as $N \rightarrow \infty$. As will be shown, this type of breaks will lead to inconsistency of the estimated factors and the overestimation of $r$. Assumption 1(b), in turn, provides the definition of small breaks which are characterized as being of order $1 / \sqrt{N T}$, so the true factor space and $r$ can be both consistently estimated under such breaks. If we only focus on the consistency of the estimated factors, the definition of small breaks can be relaxed to $\left\|\eta_{i}\right\|=O\left(N^{\delta_{1}} T^{\delta_{2}}\right)$ with $\delta_{1}, \delta_{2} \leq 0$ and $\delta_{1}+\delta_{2} \neq 0$. As shown by Theorem 1 of Bates et al. (2013), the estimated factors using PCA are still consistent for breaks with such sizes, although at a slower convergence rate than $\min (\sqrt{N}, \sqrt{T})$. However, as argued in the Introduction, we are concerned with the number of factors as well, and such breaks will possibly lead to an overestimated number of factors. Therefore, our assumption for small breaks is more stringent.

Remark 1. Bates et al. (2013) considers instabilities in the factor loadings such that: $A_{t}=A+h_{N T} * u_{t}$, where $h_{N T}$ is a scalar which depends on $N$ and $T$, and $u_{t}$ is a vector of possibly random disturbances. They propose conditions about $h_{N T}$ and $u_{t}$ under which the PCA estimator of the factors are still consistent. In the case of structural breaks, they assume $h_{N T}=1$ whereas $u_{t}$ is vector of $O(1)$ elements (say $u_{t}=\Delta \mathbf{1}(t>\tau)$ where $\Delta=$ $\left.\left(\Delta_{1}, \ldots, \Delta_{N}\right)^{\prime}\right)$ that do not depend on $N$ or $T$ after the break date $\tau$. Unlike our definitions, they characterize the size of breaks as the number of nonzero elements in $\Delta$, i.e., the number of variables having breaks in their factor loadings. For the consistency of the estimated factors, their conditions allow at most $N^{\delta}(\delta<1)$ variables to have breaks $\left(\sum_{i=1}^{N}\left\|\Delta_{i}\right\|=O\left(N^{\delta}\right)\right)$. By contrast, for the consistency of the estimated number of factors using BN's (2002) method, only a fixed number of variables are allowed to have breaks $\left(\sum_{i=1}^{N}\left\|\Delta_{i}\right\|=O(1)\right)$, when $N / T$ converges to some nonzero constant.
To compare these conditions with our definitions, notice that when $N^{\delta}$ variables are allowed to have breaks, we have that $\left\|N^{-1} \sum_{i=1}^{N} \Delta_{i} \Delta_{i}^{\prime}\right\| \leq N^{-1} \sum_{i=1}^{N}\left\|\Delta_{i}\right\|^{2}=O\left(N^{\delta-1}\right)=o(1)$ when $\delta<1$. On the other hand, our Assumption 1(a) for big breaks implies that $\left\|\frac{1}{N} \sum_{i=1}^{N} \lambda_{i} \lambda_{i}^{\prime}\right\|$ converges to a positive constant. In this sense, the big breaks defined in our paper have larger sizes than those considered in Bates et al. (2013), under which the estimated factors are proved to be consistent. As for the small breaks, our Assumption 1(b) implies that $\sum_{i=1}^{N}\left\|\eta_{i}\right\|=O(1)$ when $N$ and $T$ have the same order, similar to the conditions of Bates et al. (2013) which also allows the number of factors to be consistently estimated.

To investigate the influence of breaks on the number and estimation of factors, some further assumptions need to be imposed. To achieve consistent notation with the previous literature in the discussion of these assumptions, we follow the presentation of BN (2002) and Bai (2003) with a few slight modifications.

Assumption 2. Factors: $E\left(F_{t}\right)=0, E\left\|F_{t}\right\|^{4}<\infty$, $(N T)^{-4} E\left\|F_{t}\right\|^{8}<$ $\infty, T^{-1} \sum_{t=1}^{T} F_{t} F_{t}^{\prime} \stackrel{p}{\rightarrow} \Sigma_{F}$ and $T^{-1} \sum_{t=1}^{\tau} F_{t} F_{t}^{\prime} \stackrel{p}{\rightarrow} \pi^{*} \Sigma_{F}$ as $T \rightarrow \infty$ for some positive definite matrix $\Sigma_{F}$ where $\pi^{*}=\lim _{T \rightarrow \infty} \frac{\tau}{T}$.

Assumption 3. Factor Loadings: $\left\|\alpha_{i}\right\| \leq \bar{\alpha}<\infty$, and $N^{-1} A^{\prime} A \rightarrow$ $\Sigma_{A}, N^{-1} \Gamma^{\prime} \Gamma \rightarrow \Sigma_{\Gamma}$ as $N \rightarrow \infty$ for some positive definite matrix $\Sigma_{A}$ and $\Sigma_{\Gamma}$, where $\Gamma=\left[\begin{array}{ll}A & \Lambda\end{array}\right]$.

Assumption 4. Idiosyncratic Errors: The error terms $e_{t}$, the factors $F_{t}$ and the loadings $A$ satisfy the Assumptions A, B, C, D, E, F1, F2 of Bai (2003).

Assumption 5. Independence of Factors and Idiosyncratic Errors: $\left[F_{t}\right]_{t=1}^{T}$ and $\left[e_{t}\right]_{t=1}^{T}$ are two mutually independent groups, and $1 / \sqrt{T} \sum_{t=1}^{T} F_{t} e_{i t}=O_{p}(1)$ for each $i=1, \ldots, N$.

Assumptions 3 and 4 are standard in the literature on FM allowing for weak cross-sectional and temporal correlations between the errors. In our specific setup, Assumption 3 excludes the case where a new (old) factor appears (disappears) after the break since this event would imply that $\Sigma_{\Gamma}$ becomes singular. However, this is not restrictive since we could always envisage any potential factor as having non-zero, albeit small, loadings in either of the relevant subsamples. Assumption 2, in turn, is a new one. Since factors and factor loadings cannot be separately identified, we have to assume that DGPs with breaks in the loadings, which can be reabsorbed by transformations of the factors, should not be included in the alternative. In Section 4.4, we will discuss how to differentiate between breaks in the factor loadings and breaks in the dynamics of the factors. Different factors are allowed to be correlated at all leads and lags. Assumption 5 on the independence among the different groups is stronger than the usual assumptions made by BN (2002). Notice, however, that we could have also assumed some dependence between these groups and then impose some restrictions on this dependence when necessary. Yet, this would complicate the proofs without essentially altering the insight underlying our approach. Thus, for the sake of simplicity, we assume them to be independent in the sequel.

\section{The effects of structural breaks}

In this section, we study the effects of structural breaks on the estimation of both the number of factors based on the information criteria (IC, henceforth) proposed by BN (2002) and the factors themselves through PCA. Our main finding is that, in contrast to Stock and Watson's $(2002,2009)$ consistency result for the true 
factor space under small breaks, the factor space estimated by PCA is inconsistent, and that the number of factors tends to be overestimated under big breaks.

\subsection{The estimation of factors}

Let us rewrite model (4) with $k_{1}$ big breaks and $k_{2}$ small breaks in the more compact form:

$X_{t}=A F_{t}+\Lambda G_{t}^{1}+\epsilon_{t}$

where $\epsilon_{t}=H G_{t}^{2}+e_{t}$. The idea is to show that the new error terms $\epsilon_{t}$ still satisfy the necessary conditions for (5) being a standard FM with new factors $F_{t}^{*}=\left[\begin{array}{ll}F_{t}^{\prime} & G_{t}^{1^{\prime}}\end{array}\right]^{\prime}$ and new factor loadings $\left[\begin{array}{ll}A & \Lambda\end{array}\right]$.

Let $\bar{r}$ be the selected number of factors, either chosen by some prior knowledge or by using some consistent estimator such as the IC of BN (2002). Notice that $\bar{r}$ is not necessarily equal to $r$. Let $\hat{F}$ be $\sqrt{T}$ times the $\bar{r}$ eigenvectors corresponding to the $\bar{r}$ largest eigenvalues of the matrix $X X^{\prime}$, where the $T \times N$ matrix $X=$ $\left[\bar{X}_{1}, \bar{X}_{2}, \ldots, \bar{X}_{T}\right]^{\prime}, \bar{X}_{t}=\left[X_{t 1}, X_{t 2}, \ldots, X_{t N}\right]^{\prime}, \hat{F}=\left[\hat{F}_{1}, \hat{F}_{2}, \ldots, \hat{F}_{T}\right]^{\prime}$. Then we have:

Proposition 1. For any fixed $\bar{r}\left(1 \leq \bar{r} \leq r+k_{1}\right)$, under Assumptions $1-5$, there exists a full rank $\bar{r} \times\left(r+k_{1}\right)$ matrix $D$ and $\delta_{N, T}=\min \{\sqrt{N}, \sqrt{T}\}$ such that:

$\hat{F}_{t}=D F_{t}^{*}+O_{p}\left(\delta_{N, T}^{-1}\right) \quad$ for $t=1,2, \ldots, T$.

This result implies that $\hat{F}_{t}$ estimates consistently the space of the new factors, $F_{t}^{*}$, but not the space of the original factors, $F_{t}$.

Let us next consider two cases. First, when $k_{1}=0$ (no big breaks), we have that $G_{t}^{1}=0$, and $F_{t}^{*}=F_{t}$, so that (6) becomes

$\hat{F}_{t}=D F_{t}+O_{p}\left(\delta_{N, T}^{-1}\right)$

for a $\bar{r} \times r$ matrix $D$ of full rank. This just trivially replicates the well-known consistency result of BN (2002). ${ }^{3}$

Secondly, in the more interesting case where $k_{1}>0$ (big breaks exist), we can rewrite (6) as

$\hat{F}_{t}=\left[D_{1} D_{2}\right]\left(\begin{array}{c}F_{t} \\ G_{t}^{1}\end{array}\right)+o_{p}(1)=D_{1} F_{t}+D_{2} G_{t}^{1}+o_{p}(1)$

where the $\bar{r} \times\left(r+k_{1}\right)$ matrix $D$ is partitioned into the $\bar{r} \times r$ matrix $D_{1}$ and the $\bar{r} \times k_{1}$ matrix $D_{2}$. Note that, by the definition of $G_{t}, G_{t}^{1}=0$ for $t=1,2, \ldots, \tau$, and $G_{t}^{1}=F_{t}^{1}$ for $t=\tau+1, \ldots, T$, where $F_{t}^{1}$ is the $k_{1} \times 1$ sub-vector of $F_{t}$ that is subject to big breaks in their loadings. Therefore ( 8 ) can be expressed as:

$\hat{F}_{t}=D_{1} F_{t}+o_{p}(1)$ for $t=1,2, \ldots, \tau$,

$\hat{F}_{t}=D_{2}^{\dagger} F_{t}+o_{p}(1)$ for $t=\tau+1, \ldots, T$

where $D_{2}^{\dagger}=D_{1}+\left[\begin{array}{ll}D_{2} & 0\end{array}\right], 0$ is a $\bar{r} \times\left(r-k_{1}\right)$ zero matrix, and $D_{2} \neq 0$ when $\bar{r}>r$. Hence, since $D_{1} \neq D_{2}^{\dagger}$, this implies that, in contrast to small breaks where $D_{2}$ tends to 0 due to the local-tozero properties of the elements of $H$ (see Assumption 1(b)), under big breaks the estimated factors $\hat{F}$ will not be consistent for the space of the true factors $F$. Accordingly, as will be discussed below, imposing a priori the number of estimated factors to be used as predictors or explanatory variables in standard factor-augmented models may lead to misleading results.

\footnotetext{
3 Notice that, for the estimator $\hat{F}$ defined here, $\bar{r}$ has to be smaller or equal to $r$ for (7) to hold.
}

To illustrate the consequences of having big breaks in the factor loadings, consider the following simple Factor Augmented Regression (FAR) model (see BN, 2006a):

$y_{t}=a^{\prime} F_{t}+b^{\prime} W_{t}+u_{t}, \quad t=1,2, \ldots, T$

where $W_{t}$ is a small set of observable variables and the $r \times 1$ vector $F_{t}$ contains the $r$ common factors driving a large panel data set $X_{i t}$ $(i=1,2, \ldots, N ; t=1,2, \ldots, T)$ which excludes both $y_{t}$ and $W_{t}$. The parameters of interest are the elements of vector $b$ while $F_{t}$ is included in (11) to control for potential endogeneity arising from omitted variables. Since we cannot identify $F_{t}$ and $a$, only the product $a^{\prime} F_{t}$ is relevant. Suppose that there is a big break at date $\tau$. From (9) and (10), we can rewrite (11) as:

$y_{t}=\left(a^{\prime} D_{1}^{-}\right)\left(D_{1} F_{t}\right)+b^{\prime} W_{t}+u_{t}$ for $t=1,2, \ldots, \tau$,

$y_{t}=\left(a^{\prime} D_{2}^{\dagger-}\right)\left(D_{2}^{\dagger} F_{t}\right)+b^{\prime} W_{t}+u_{t}$ for $t=\tau+1, \ldots, T$

where $D_{1}^{-} D_{1}=D_{2}^{\dagger-} D_{2}=I_{r}$, or equivalently

$y_{t}=a_{1}^{\prime} \hat{F}_{t}+b^{\prime} W_{t}+\tilde{u}_{t}$ for $t=1,2, \ldots, \tau$,

$y_{t}=a_{2}^{\prime} \hat{F}_{t}+b^{\prime} W_{t}+\tilde{u}_{t} \quad$ for $t=\tau+1, \ldots, T$

where $a_{1}^{\prime}=a^{\prime} D_{1}^{-}$and $a_{2}^{\prime}=a^{\prime} D_{2}^{\dagger-}$, and $\tilde{u}_{t}=u_{t}+o_{p}(1)$.

If the number of factors is a priori known to be $\bar{r}=r$, then $D_{1}^{-}=D_{1}^{-1}, D_{2}^{\dagger-}=D_{2}^{\dagger-1}$. If we assume $D_{1} \neq D_{2}^{\dagger}$, it follows that $D_{1}^{-1} \neq D_{2}^{\dagger-1}$ and thus $a_{1} \neq a_{2}$. Therefore, using the indicator function $\mathbf{1}(t>\tau),(12)$ and (13) can be rewritten as

$y_{t}=a_{1}^{\prime} \hat{F}_{t}+\left(a_{2}-a_{1}\right)^{\prime} \hat{F}_{t} \mathbf{1}(t>\tau)+b^{\prime} W_{t}+\tilde{u}_{t}$,

$t=1,2, \ldots, T$.

A straightforward implication of the previous result is that if we were to impose the number of factors, on a priori ground, therefore ignoring the set of regressors $\hat{F}_{t} \mathbf{1}(t>\tau)$ in (14), in general the estimation of $b$ will become inconsistent due to omitted variables.

Remark 2. Interestingly, there are many examples in the literature where, for theoretical or practical reasons, the number of factors is imposed as a prior. For example, to name a few, a single common factor representing a global effect is assumed in the wellknown study by Bernanke et al. (2005) on measuring the effects of monetary policy in Factor Augmented VAR (FAVAR) models, as well as in the risk analysis in portfolios of corporate debt by Gagliardini and Gouriéroux (2011) where a single factor is supposed to capture a latent macro-variable. Likewise, two factors are a priori imposed by Rudebusch and Wu (2008) in their macro-finance model. Notice that a similar argument will render inconsistent the impulse response functions in FAVAR models where the regressand in (11) becomes $y_{t+1}=\left(F_{t+1}, W_{t+1}\right)^{\prime}$.

Remark 3. Alternatively, if the number of factors is not a priori imposed and it is estimated by the IC of BN (2002), we will show in the next section (Proposition 2) that the estimated number of factors will tend to $r+k_{1}$ as the sample size grows. In this case, $D_{1}$ and $D_{2}^{\dagger}$ are $\left(r+k_{1}\right) \times r$ matrices, and by the definitions of $D_{1}$ and $D_{2}^{\dagger}$, it is easy to show that we can always find a $r \times\left(r+k_{1}\right)$ matrix $D^{-}=D_{1}^{-}=D_{2}^{\dagger-}$ such that $D^{-} D_{1}=D^{-} D_{2}^{\dagger}=I_{r}$. If we define

$\bar{a}^{\prime}=a^{\prime} D^{-}$,

then $a_{1}^{\prime}=a_{2}^{\prime}=\bar{a}^{\prime}$ so that (12) and (13) can be rewritten as

$y_{t}=\bar{a}^{\prime} \hat{F}_{t}+b^{\prime} W_{t}+\tilde{u}_{t}, \quad t=1,2, \ldots, T$,

so that the estimation of (11) will not be affected by the estimated factors under big breaks if $\bar{r}=r+k_{1}$. 
Remark 4. Yet, even in this case, the factors themselves may be the direct subject of interest and thus their interpretation can have important implications for structural analysis. For example, a large body of empirical research in financial economics is concerned with identifying the factors that determine asset returns. ${ }^{4}$ However, the existence of big breaks may hamper this identification procedure. For instance, if $\widehat{r}=2$, a relevant question would be: are there two genuine factors or one factor and one break? Our testing procedure provides a useful tool to disentangle these two cases (see Section 4.4 below).

Another area where our testing approach could be useful is in applications where the estimated factors are modeled in a VAR in order to identify the structural shocks driving them (see e.g. Charnavoki and Dolado, 2012). This identification becomes more difficult as the number of factors increases. For this reason, it is very important to determine whether the selection of a large number of factors is due to having several genuine factors or to a break affecting some of them. The insight is that instead of having to identify $r+k_{1}$ shocks we would only have to identify $r$.

Summing up, the use of estimated factors as the true factors when assuming that the number of factors is a priori known will lead to inconsistent estimates in a FAR under big breaks. As a simple remedy, $\hat{F}_{t} \mathbf{1}(t>\tau)$ should be added as regressors when big breaks are detected and the break date is located. Alternatively, without pretending to know a priori the true number of factors, the estimation of FAR will be robust to the estimation of factors under big breaks if the number of factors is chosen according to some consistent estimator. Yet, this may hinder the correct interpretation of the estimated factors in terms of observables. As a result, in order to run regression (16), our advice is to avoid imposing the number of factors a priori, unless a formal test of big breaks is implemented. We will illustrate these points in Section 5 by means of simulations in a typical forecasting exercise where the predictors are common factors estimated by PCA.

\subsection{The estimated number of factors}

$\mathrm{BE}$ (2011) have previously argued that the presence of structural breaks in the factor loadings may lead to the overestimation of the number of factors. Yet, since they do not provide a formal proof of this result, we proceed to fill this gap.

Let $\hat{r}$ be the estimated number of factors in (5) using the IC proposed by BN (2002), and $\vartheta_{N T}^{1}$ be the largest eigenvalue of $(N T)^{-1} \sum_{t=1}^{T} e_{t} e_{t}^{\prime}$. Then, the following result holds:

Proposition 2. Suppose $\vartheta_{N T}^{1}=O_{p}\left(\delta_{N T}^{-2}\right)$ and Assumptions 1-5 hold, then:

$\lim _{N, T \rightarrow \infty} \mathbb{P}\left[\hat{r}=r+k_{1}\right]=1$.

Again, absent big breaks $\left(k_{1}=0\right)$, this result trivially replicates Theorem 2 of BN (2002). However, under big breaks $\left(k_{1}>0\right)$, their IC will overestimate the number of original factors by the number of big breaks $\left(0<k_{1} \leq r\right)$ because, as shown above, a FM with this type of break admits a representation without a break but with more factors.

In sum, when we use PCA to estimate the factor space and the IC of BN (2002) to estimate the number of factors, the small breaks can be safely ignored, while the big breaks will lead to the inconsistencies of $\hat{F}_{t}$ and $\hat{r}$.

\footnotetext{
4 Chen et al. (1986) and Shanken and Weinstein (2006) are good illustrations of attempts to interpret the underlying forces in the stock market developments in terms of some observed macro variables.
}

\section{Testing for structural breaks}

\subsection{Hypotheses of interest and test statistics}

Our goal here is to develop a test for big breaks. As mentioned above, if we were to follow the usual approach in the literature to test for structural breaks, we would consider the following null and alternative hypotheses in (1) and (2): $H_{0}: A=B$ vs. $H_{1}: A \neq B$. However, this standard formulation faces two problems. First, if only small breaks occur, the estimation and inference based on PAC are not affected. Thus, we can ignore these breaks. Secondly, and foremost, since $A$ and $B$ are $N \times r$ matrices, we would face an infinite-dimensional parameter problem as $N$ grows if we were to consider differences in all their individual elements. To solve the first problem, we focus only on big breaks and consider $H_{0}$ : $k_{1}=0$ vs. $H_{1}: k_{1}>0$, where the new null and alternative hypotheses correspond to the cases where there are no big breaks (yet there may be small breaks) and there is at least one big break, respectively.

Relying upon the discussion in Section 3.1 about the inconsistency of $\widehat{F}$ for the space of the true factors $F$ when big breaks occur, our strategy to circumvent the second problem is to focus on how the dependence properties of the $\bar{r}$ estimated factors (using the whole sample) change before and after the potential break date. Since, in line with the standard assumption in FM, the number of true factors $(r)$ is considered to be invariant to the sample size, our previous result in Proposition 2 ensures that $r+k_{1}$, with $k_{1} \leq r$, is finite-dimensional.

To test the above null hypothesis, we consider the following two-step procedure:

1. In the first step, the number of factors to estimate, $\bar{r}$, is determined and $\bar{r}$ common factors $\left(\hat{F}_{t}\right)$ are estimated by PCA.

2 . In the second step, we consider the following linear regression of one of the estimated factors on the remaining $\bar{r}-1$ ones. For example, using the first factor as the regressand, this leads to the regression:

$\hat{F}_{1 t}=c_{2} \hat{F}_{2 t}+\cdots+c_{\bar{r}} \hat{F}_{\bar{r} t}+u_{t}=c^{\prime} \hat{F}_{-1 t}+u_{t}$

where $\hat{F}_{-1 t}=\left[\hat{F}_{2 t}, \ldots, \hat{F}_{\bar{r} t}\right]^{\prime}$ and $c=\left[c_{2}, \ldots, c_{\bar{r}}\right]^{\prime}$ are $(\bar{r}-1) \times 1$ vectors. Then we test for a structural break of $c$ in the above regression. If a structural break is detected, then we reject $H_{0}$ : $k_{1}=0$; otherwise, we cannot reject the null of no big breaks.

Remark 5. In the first stage, we have recommended to choose $\bar{r}$ as some consistent estimator of $r$ to obtain the best size and power properties. Although our Proposition 2 is based on the $\hat{r}$ estimated by the IC of BN (2002), one can also use other procedures to consistently estimate $r$. For example, Onatski $(2009,2010)$, and Ahn and Horenstein (2013) show that their methods have better finite sample properties than BN (2002) especially when the errors are cross sectionally correlated. That being said, as will be discussed below, one important feature of our tests is that they do not rely on the correct estimation of $r$. In the second step, although there are many methods of testing for breaks in a simple linear regression model, we follow Andrews (1993) to define the LM and Wald tests when the possible break date is assumed to be known, and their Sup-type versions when there is no prior knowledge about it. Moreover, since the LM, Wald and LR test statistics have the same asymptotic distribution under the null, we focus on the first two because they are computationally simpler.

Define $D^{*}=V^{-1 / 2} \Upsilon^{\prime} \Sigma_{A}^{1 / 2}$ as the limit of the matrix $D$ in Eq. (7), where $V=\operatorname{diag}\left(v_{1}, v_{2}, \ldots, v_{r}\right), v_{1}>v_{2}>\cdots>$ $v_{r}$ are the eigenvalues of $\Sigma_{A}^{1 / 2} \Sigma_{F} \Sigma_{A}^{1 / 2}$, and $\Upsilon$ is the corresponding eigenvector matrix (see Bai, 2003). Define $\mathcal{F}_{1 t}=D_{1}^{*} F_{t}$ and 
$\mathcal{F}_{-1 t}=D_{-1}^{*} F_{t}$, where $D_{1}^{*}$ is the first row of $D^{*}$ and $D_{-1}^{*}$ is the matrix containing the second to last rows of $D^{*}$. Finally, let the $(r-1) \times(r-1)$ matrix $S=\lim \operatorname{Var}\left(\frac{1}{T} \sum_{t=1}^{T} \mathcal{F}_{-1 t} \mathcal{F}_{1 t}\right)$.

Following Andrews (1993), the LM test statistic is then defined as follows:

$$
\mathcal{L}(\bar{\pi})=\frac{1}{\bar{\pi}(1-\bar{\pi})}\left(\frac{1}{\sqrt{T}} \sum_{t=1}^{\tau} \hat{F}_{-1 t} \hat{u}_{t}\right)^{\prime} \hat{S}^{-1}\left(\frac{1}{\sqrt{T}} \sum_{t=1}^{\tau} \hat{F}_{-1 t} \hat{u}_{t}\right)
$$

where $\bar{\pi}=\tau / T, \tau$ is a pre-assumed date for the potential break, $\hat{u}_{t}$ is the residual in the OLS regression (17), which by construction equals $\hat{F}_{1 t}$, and $\hat{S}$ is a consistent estimator of $S .^{5}$

The corresponding Sup-LM statistic is defined as:

$$
\begin{aligned}
\mathcal{L}(\Pi)= & \sup _{\pi \in \Pi} \frac{1}{\pi(1-\pi)}\left(\frac{1}{\sqrt{T}} \sum_{t=1}^{[T \pi]} \hat{F}_{-1 t} \hat{u}_{t}\right)^{\prime} \hat{S}^{-1} \\
& \times\left(\frac{1}{\sqrt{T}} \sum_{t=1}^{[T \pi]} \hat{F}_{-1 t} \hat{u}_{t}\right)
\end{aligned}
$$

where $\Pi$ is any set whose closure lies in $(0,1)$.

Similarly, the Wald and Sup-Wald test statistics can be constructed as:

$$
\mathcal{L}^{*}(\bar{\pi})=\bar{\pi}(1-\bar{\pi}) \cdot T\left(\hat{c}_{1}(\bar{\pi})-\hat{c}_{2}(\bar{\pi})\right)^{\prime} \hat{S}^{-1}\left(\hat{c}_{1}(\bar{\pi})-\hat{c}_{2}(\bar{\pi})\right)
$$

and

$$
\begin{aligned}
\mathcal{L}^{*}(\Pi)= & \sup _{\pi \in \Pi} \pi(1-\pi) \cdot T\left(\hat{c}_{1}(\pi)-\hat{c}_{2}(\pi)\right)^{\prime} \hat{S}^{-1} \\
& \times\left(\hat{c}_{1}(\pi)-\hat{c}_{2}(\pi)\right)
\end{aligned}
$$

where $\hat{c}_{1}(\pi)$ and $\hat{c}_{2}(\pi)$ are OLS estimates of $c$ using subsamples before and after the break point : $[T \pi]{ }^{6}$

To illustrate why our two-step testing procedure is able to detect the big breaks, it is useful to consider a simple example where $r=1, k_{1}=1$ (one common factor and one big break). Then (5) becomes:

$X_{t}=A f_{t}+B g_{t}+e_{t}$

where $g_{t}=0$ for $t=1, \ldots, \tau$, and $g_{t}=f_{t}$ for $t=\tau+1, \ldots, T$. By Proposition 2, we will tend to get $\hat{r}=2$ in this case. Suppose now that we estimate 2 factors $(\bar{r}=2)$. Then, by Proposition 1 , we have:

$$
\left(\begin{array}{l}
\hat{f}_{1 t} \\
\hat{f}_{2 t}
\end{array}\right)=D\left(\begin{array}{l}
f_{t} \\
g_{t}
\end{array}\right)+o_{p}(1)
$$

where $D=\left(\begin{array}{ll}d_{1} & d_{2} \\ d_{3} & d_{4}\end{array}\right)$ is a non-singular matrix. By the definition of $g_{t}$ we have:

$$
\begin{aligned}
& \hat{f}_{1 t}=d_{1} f_{t}+o_{p}(1) \quad \hat{f}_{2 t}=d_{3} f_{t}+o_{p}(1) \quad \text { for } t=1, \ldots, \tau, \\
& \hat{f}_{1 t}=\left(d_{1}+d_{2}\right) f_{t}+o_{p}(1) \quad \hat{f}_{2 t}=\left(d_{3}+d_{4}\right) f_{t}+o_{p}(1) \\
& \quad \text { for } t=\tau+1, \ldots, T
\end{aligned}
$$

\footnotetext{
5 See Appendix A.3 for discussions on the estimation of $S$.

6 We can also construct the Wald test as $T\left(\hat{c}_{1}(\bar{\pi})-\hat{c}_{2}(\bar{\pi})\right)^{\prime}\left(\frac{\hat{S_{1}}}{\bar{\pi}}+\right.$ $\left.\frac{\hat{S}_{2}}{(1-\bar{\pi})}\right)^{-1}\left(\hat{c}_{1}(\bar{\pi})-\hat{c}_{2}(\bar{\pi})\right)$ and the Sup-Wald test similarly, where $\hat{S}_{1}$ and $\hat{S}_{2}$ are estimates of $S$ using subsamples. Yet, in all our simulations, the results based on these two methods are very similar. Therefore, for brevity, we focus on the ones obtained using the full sample estimation of $S$, as in (20) and (21).
}

which imply that:

$\hat{f}_{1 t}=\frac{d_{1}}{d_{3}} \hat{f}_{2 t}+o_{p}(1)$ for $t=1, \ldots, \tau$,

$\hat{f}_{1 t}=\frac{d_{1}+d_{2}}{d_{3}+d_{4}} \hat{f}_{2 t}+o_{p}(1) \quad$ for $t=\tau+1, \ldots, T$.

Thus, we can observe that the two estimated factors are linearly related and that the coefficients $\frac{d_{1}}{d_{3}}$ and $\frac{d_{1}+d_{2}}{d_{3}+d_{4}}$ before and after the break date must be different due to the non-singularity of the $D$ matrix. As a result, regressing one of the estimated factors on the other and testing for a structural break in this regression, we should reject the null of no big break. In the case where $d_{3}=0$, the above argument fails. But since $d_{1}$ and $d_{4}$ are non-zeros (otherwise $D$ will have reduced-rank), the estimated slope in the first subsample will diverge while it will converge to some bounded number in the second subsample. Therefore our test also has power in this case. ${ }^{7}$

Likewise, if the break date $\tau$ is not a priori assumed to be known, the Sup-type tests will yield a natural estimate of $\tau$ at the date when the test reaches its maximum value. In what follows, we derive the asymptotic distribution of the test statistics (18)-(21) under the null hypothesis, as well as extend the intuition behind this simple example to the more general case to show that our tests have power against relevant alternatives.

\subsection{Limiting distributions under the null hypothesis}

Since in most applications the number of factors is estimated by means of BN's (2002) IC, and it converges to the true one under the null hypothesis of no big break, we start with the most interesting case where $\bar{r}=r$. To derive the asymptotic distributions of the LM and Wald statistics, we adopt the following additional assumptions:

Assumption 6. $\sqrt{T} / N \rightarrow 0$ as $N \rightarrow \infty$ and $T \rightarrow \infty$.

Assumption 7. $\left\{F_{t}\right\}$ is a stationary and ergodic sequence, and $\left\{F_{i t} F_{j t}-E\left(F_{i t} F_{j t}\right), \Omega_{t}\right\}$ is an adapted mixingale with $\gamma_{m}$ of size -1 for $i, j=1,2, \ldots, r$, that is:

$\sqrt{E\left(E\left(Y_{i j, t} \mid \Omega_{t-m}\right)^{2}\right)} \leq c_{t} \gamma_{m}$

where $Y_{i j, t}=F_{i t} F_{j t}-E\left(F_{i t} F_{j t}\right), \Omega_{t}$ is a $\sigma$-algebra generated by the information at time $t, t-1, \ldots,\left\{c_{t}\right\}$ and $\left\{\gamma_{m}\right\}$ are non-negative sequences and $\gamma_{m}=O\left(m^{-1-\delta}\right)$ for some $\delta>0$.

Assumption 8. $\sup _{\pi \in[0,1]}\left\|\frac{1}{\sqrt{N T}} \sum_{t=1}^{T \pi} \sum_{i=1}^{N} \alpha_{i} F_{t}^{\prime} e_{i t}\right\|^{2}=O_{p}(1)$.

Assumption 9. $\|\hat{S}-S\|=o_{p}(1)$, and $S$ is a $(r-1) \times(r-1)$ symmetric positive definite matrix.

Assumption 10. The eigenvalues of the $r \times r$ matrix $\Sigma_{A} \Sigma_{F}$ are distinct.

Assumptions 6 and 8 are required to bound the estimation errors of $\hat{F}_{t}$, while Assumption 7 is needed to derive the weak convergence of the test statistics using the Functional Central Limit Theorem (FCLT). Assumption 10 corresponds to Assumption G of Bai (2003), which is required for $D \stackrel{p}{\rightarrow} D^{*}$.

Note that these assumptions are not restrictive. Assumption 6 allows $T$ to be $O\left(N^{1+\delta}\right)$ for $-1<\delta<1$. As for Assumption 7, it allows us to consider a quite general class of linear processes for

\footnotetext{
7 This is the case for the Wald test but may not be true for the LM test, because our Wald test is directly built upon the difference between the estimated coefficients.
} 
the factors: $F_{i t}=\sum_{k=0}^{\infty} \theta_{i k} v_{i, t-k}$, where $v_{t}=\left[v_{1 t} \ldots v_{r t}\right]^{\prime}$ are i.i.d. with zero means, and $\operatorname{Var}\left(v_{i t}\right)=\sigma_{i}^{2}<\infty$. In this case, it can be shown that:

$\sqrt{E\left(E\left(Y_{i j, t} \mid \Omega_{t-m}\right)^{2}\right)} \leq \sigma_{i} \sigma_{j}\left(\sum_{k=m}^{\infty}\left|\theta_{i k}\right|\right)\left(\sum_{k=m}^{\infty}\left|\theta_{j k}\right|\right)$

for which it suffices that

$\left(\sum_{k=m}^{\infty}\left|\theta_{i k}\right|\right)=O\left(m^{-1 / 2-\delta}\right)$

for some $\delta>0$, which is satisfied for a large class of ARMA processes. Assumption 8 is similar to Assumption F.2 of Bai (2003), which involves zero-mean random variables. Finally, a consistent estimate of $S$ can be calculated by a HAC estimator such as Newey and West's (1987) estimator with a Bartlett kernel, which is the one used in our simulations below. ${ }^{8}$

Let $\stackrel{d}{\rightarrow}$ " denote convergence in distribution, then:

Theorem 1. Under the null hypothesis $H_{0}: k_{1}=0$ and Assumptions $1-10$ as $N, T \rightarrow \infty$, we have that both the LM and Wald tests verify

$\mathcal{L}(\bar{\pi}), \mathscr{L}^{*}(\bar{\pi}) \stackrel{d}{\rightarrow} \chi^{2}(r-1)$

where $\bar{\pi}=\tau / T$ for a given $\tau$; and

$$
\begin{aligned}
\mathcal{L}(\Pi), \mathcal{L}^{*}(\Pi) \stackrel{d}{\rightarrow} & \sup _{\pi \in \Pi}\left(W_{r-1}(\pi)-\pi W_{r-1}(1)\right)^{\prime} \\
& \times\left(W_{r-1}(\pi)-\pi W_{r-1}(1)\right) /[\pi(1-\pi)]
\end{aligned}
$$

for any set $\Pi$ whose closure lies in $(0,1)$, where $w_{r-1}(\cdot)$ is a $r-1$ vector of independent Brownian Motions on $[0,1]$ restricted to $\Pi$. (1993)

The critical values for the Sup-type test are provided in Andrews

Remark 6. It is easy to show that Theorem 1 still holds when $\bar{r}<r$. However, when $\bar{r}>r$, the properties of $\hat{F}_{\bar{r} t}$ are unknown since the $\bar{r}$ th eigenvectors of $X X^{\prime}$ may be related to the properties of $e_{t}$. Thus, the asymptotic distribution cannot be derived in a similar way. Yet, as the simulations in Section 5 show, in such an instance Theorem 1 still provides a reasonably good approximation for the distributions of our test statistics in finite samples. Moreover, the case where $\bar{r}>r$ can be avoided if, instead of relying on a priori choice of $\bar{r}$, practitioners use BN's (2002) IC or other consistent estimators of $r$, in line with Proposition 2.

\subsection{Performance of the tests under the alternative hypothesis}

We now extend the insight of the simple example considered in Section 4.1 to show that, under the alternative hypothesis $\left(k_{1}>0\right)$, the linear relationship between the estimated factors changes at time $\tau$, so that the proposed tests are able to detect big breaks.

Assuming that $r<\bar{r} \leq r+k_{1}$, then the matrix $D_{1}$ and $D_{2}^{\dagger}$ in (9) and (10) become $\bar{r} \times r$ matrices. Notice that since $\bar{r}>r$ we can always find $\bar{r} \times 1$ vectors $\rho_{1}$ and $\rho_{2}$ which belong to the null spaces of $D_{1}^{\prime}$ and $D_{2}^{\dagger^{\prime}}$ separately, that is, $\rho_{1}^{\prime} D_{1}=0$ and $\rho_{2}^{\prime} D_{2}^{\dagger}=0$. Hence, premultiplying both sides of (9) and (10) by $\rho_{1}^{\prime}$ and $\rho_{2}^{\prime}$ leads to:

$\rho_{1}^{\prime} \hat{F}_{t}=o_{p}(1) \quad t=1,2, \ldots, \tau$,

$\rho_{2}^{\prime} \hat{F}_{t}=o_{p}(1) \quad t=\tau+1, \ldots, T$

8 Though not reported, other estimators, like those based on Parzen kernels, yield similar results in our simulations about the size and power properties of the LM and Wald tests. which, after normalizing one of the elements of $\rho_{1}$ and $\rho_{2}$ (e.g., the first one) to be 1 , implies that:

$\hat{F}_{1 t}=\hat{F}_{-1 t}^{\prime} \rho_{1}^{*}+o_{p}(1) \quad t=1,2, \ldots, \tau$,

$\hat{F}_{1 t}=\hat{F}^{\prime}{ }_{-1 t} \rho_{2}^{*}+o_{p}(1) \quad t=\tau+1, \ldots, T$,

where $\hat{F}^{\prime}{ }_{-1 t}=\left[\hat{F}_{2 t}, \ldots, \hat{F}_{\bar{r} t}\right]$ and $\rho_{1}^{*}, \rho_{2}^{*}$ are both $(\bar{r}-1) \times 1$ vectors. Next, to show that $\rho_{1}^{*} \neq \rho_{2}^{*}$, we proceed as follows. Suppose that $\gamma \in \operatorname{Null}\left(D_{1}^{\prime}\right)$ and $\gamma \in \operatorname{Null}\left(D_{2}^{\dagger^{\prime}}\right)$, then by the definitions of $D_{1}$ and $D_{2}^{\dagger}$ and by the basic properties of full-rank matrices, it holds that $\gamma \in \operatorname{Null}\left(D^{\prime}\right)$. Since $D$ is a full rank $\bar{r} \times\left(r+k_{1}\right)$ matrix and $\bar{r} \leq r+k_{1}$, then $\operatorname{Null}\left(D^{\prime}\right)=0$ and thus $\gamma=0$. Therefore, the only vector that belongs to the null space of $D_{1}$ and $D_{2}^{\dagger}$ is the trivial zero vector. Further, because the rank of the null space of $D_{1}$ and $D_{2}^{\dagger}$ is larger than 1 , we can always find two non-zero vectors such that $\rho_{1} \neq b \rho_{2}$ for any constant $b \neq 0$.

Notice that when $\bar{r} \leq r$, the rank of the null spaces of $D_{1}$ and $D_{2}^{\dagger}$ will possibly become zero. Hence, the preceding analysis does not apply in this case despite the existence of linear relationships among the estimated factors. If we regress one of the estimated factors on the others, with $\hat{\rho}_{1}$ and $\hat{\rho}_{2}$ denoting the OLS estimates of the coefficients using the two subsamples before and after the break, in general we cannot verify that plim $\hat{\rho}_{1} \neq \operatorname{plim} \hat{\rho}_{2}$.

Remark 7. One underlying assumption in the above argument is that one of the elements of $\rho_{1}$ and $\rho_{2}$ (e.g., the first ones) are different from zero. This assumption is hard to verify since the $D$ matrix depends on $\Gamma$ and $F^{*}$ in a highly nonlinear way, ${ }^{9}$ and it is not difficult to find DGPs where this assumption does not hold. This normalization issue makes it really hard to come up with a formal result on the consistency of our test. Instead, we have run a large number of simulations to study the actual power properties of our tests for various DGPs, including the ones where the first elements of $\rho_{1}$ and $\rho_{2}$ are both zeros. The general finding is that our Wald and Sup-Wald tests are very powerful for all the DGPs we have considered, while the LM and Sup-LM tests may lose power when the normalization issue arises. ${ }^{10}$ For this reason, we recommend to use the Wald test in practice. We present some of the representative simulation results in Section 5 but more results are available upon request.

Remark 8. Since our tests are based on a linear regression model, many other available methods in the literature can also be applied in our second-stage procedure. For instance, when the break date is not known as a priori, one can use the CUSUM type-test first proposed by Brown et al. (1975), and also Chen and Hong (2012) test via nonparametric regression. Thus, this flexibility allows practitioners to draw conclusions about breaks based on broader evidence than that just provided by a single test.

Remark 9. Although our tests have been designed for a single common break at date $\tau$, they should also exhibit powers against other interesting alternatives such as multiple breaks and a change in the number of factors. ${ }^{11}$

\footnotetext{
9 By the result of Bai (2003), when $\bar{r}=r+k_{1}$, the $\left(r+k_{1}\right) \times\left(r+k_{1}\right)$ matrix $D=V_{N T}^{-1}\left(\hat{F}^{*^{\prime}} F^{*} / T\right)\left(\Gamma^{\prime} \Gamma / N\right)$, where $V_{N T}$ is the diagonal matrix with the first $r+k_{1}$ eigenvalues of $X^{\prime} X / N T$.

10 Since the LM test is the one more affected by the normalization problem, an interesting issue raised by a referee is whether this problem could be avoided by using $\max _{j=1, \ldots, \bar{r}} \operatorname{LM}\left(\hat{F}_{j t}\right)$ where $\operatorname{LM}\left(\hat{F}_{j t}\right)$ is the LM test using $\hat{F}_{j t}$ as the regressand. However, because its limiting distribution is unknown under the null and likely dependent on nuisance parameters, we leave this analysis for our further research agenda.

11 See our online appendix for details.
} 


\subsection{Disentangling breaks in loadings from breaks in factors}

A potential critique for all available tests of big breaks in FM is that they cannot differentiate between breaks in factor loadings and breaks in the covariance matrix of factors. For illustrative purposes, let us consider a FM with $r=2$, where the factor loadings are constant but the covariance matrix of the factors breaks, such that: $E\left(F_{t} F_{t}^{\prime}\right)=\left(\begin{array}{ll}1 & \rho \\ \rho & 1\end{array}\right)$ for $t=1, \ldots, T / 2$, and $E\left(F_{t} F_{t}^{\prime}\right)=\left(\begin{array}{cc}1 & -\rho \\ -\rho & 1\end{array}\right)$ for $t=T / 2+1, \ldots, T$, with $\rho \notin\{0,1\}$. If we further assume $\Sigma_{A}=\lim _{N \rightarrow \infty} A^{\prime} A / N$ is a diagonal matrix, then, in view of Bai (2003), we have that $\hat{F}_{t}=F_{t}+o_{p}(1)$, where $\hat{F}_{t}$ is a $2 \times 1$ vector. In this case, regressing $\hat{F}_{1 t}$ on $\hat{F}_{2 t}$ will yield estimated coefficients close to $\rho$ and $-\rho$ before and after the break, respectively. As a result, our tests will reject the null of no big break in the loadings while the true DGP has a break in the factors.

Although the above example has been excluded by our Assumptions 2 and 7, it could well be that the factor dynamics are subject to structural breaks in practice. For instance, if the factors are interpreted as common shocks to the economy, then it is likely that their volatilities may have decreased since the beginning of 1980s, as evidenced by recent studies on the effect of the Great Moderation (see, e.g. Gali and Gambetti, 2009). Hence, for interpretational purposes, it becomes relevant to identify which is the source of breaks.

Assuming that there is only one source of instability and that one break in the FM has been detected at some date $\tau$, one can differentiate between the break in the loadings and the break in the factor dynamics by comparing the number of factors obtained with the whole sample to those in each of the subsamples split by $\tau$. To see this, notice that, absent a big break in the factor loadings, the number of factors will be consistently estimated for the whole sample and each subsamples, as long as the factors satisfy:

$$
\frac{1}{\tau} \sum_{t=1}^{\tau} F_{t} F_{t}^{\prime} \stackrel{p}{\rightarrow} \Sigma_{F}^{1}>0, \quad \text { and } \quad \frac{1}{T-\tau} \sum_{t=\tau+1}^{T} F_{t} F_{t}^{\prime} \stackrel{p}{\rightarrow} \Sigma_{F}^{2}>0 .
$$

One important observation is that, when the factors are stationary and the rejection of the null is due to big breaks in the loadings, the true number of factors, $r$, can be consistently estimated for each of the two subsamples while, for the whole sample, it will be overestimated in light of Proposition 2. Therefore, for a given data set, if $\hat{r}=2$ in the whole sample and our test rejects the null with $\bar{r}=2$, it could be that there is one factor and one big break, or two factors with changing correlation, as shown above. In the first case, the estimated numbers of factors in each of the two subsamples converge to 1 , while for the latter case it converges to 2 . Thus, these different rank properties could become the basis of our identification strategy for the source of the break.

When both breaks in the loadings and factors exist, our strategy of considering a finite dimensional linear regression still works, but the asymptotic distribution of the proposed test statistics may be different. The reason is that the estimated factors, which are consistent estimators of the true factor space under the null, may also experience breaks in their dynamics. As a result, Hansen's (2000) results imply that the asymptotic distributions of the Wald and Sup Wald tests may change if the dynamics of regressors (the estimated factors in our case) have breaks. We leave this interesting question for future research.

\section{Simulations}

In this section, we first use a simple factor-based forecasting model to illustrate the consequences of ignoring big breaks, as discussed in Section 3. Next, we study the finite sample properties of our proposed LM/Wald and Sup-LM/Wald tests. We pay special attention to the sizes and the powers when $\bar{r}>r$ since, as discussed previously, obtaining formal results for this case is very difficult. A comparison with the tests of BE (2011) and HI (2012) is also provided to illustrate the advantages of our tests in term of power. Throughout this section, the potential breaking date is considered to located at half of the sample $(\tau=T / 2)$ and is taken to be a priori known for the LM/Wald tests while $\Pi$ is chosen as $[0.15,0.85]$ for the Sup-type versions. Finally, the covariance matrix $S$ is estimated using the HAC estimator of Newey and West (1987).

\subsection{The effect of big breaks on forecasting}

In this section we consider the effect of having big breaks in a typical forecasting exercise where the predictors are estimated common factors. First, we have a large panel of data $X_{t}$ driven by the factors $F_{t}$ which are subject to a big break in the factor loadings:

$X_{t}=A F_{t} \mathbf{1}(t \leq \tau)+B F_{t} \mathbf{1}(t>\tau)+e_{t}$.

Secondly, the variable we wish to forecast $y_{t}$, which is excluded from to $X_{t}$, is assumed to be related to $F_{t}$ as follows:

$y_{t+1}=a^{\prime} F_{t}+v_{t+1}$.

We consider a DGP where $N=100, T=200, \tau=100, r=2$, $a^{\prime}=\left[\begin{array}{ll}1 & 1\end{array}\right], F_{t}$ are generated as two $\mathrm{AR}(1)$ processes with coefficients 0.8 and 0.2 , respectively, $e_{t}$ and $v_{t}$ are i.i.d. standard normal variables, and the break size is characterized by a range of mean shifts between 0 and 1 .

The following forecasting procedures are compared in our simulation:

Benchmark Forecasting: The factors $F_{t}$ are treated as observed and are used directly as predictors. The one-step-ahead forecast of $y_{t}$ is defined as $\hat{y}_{t+1 \mid t}=\hat{a}^{\prime} F_{t}$, where $\hat{a}$ is the OLS estimate of $a$ in the regression of $y_{t+1}$ on $F_{t}$.

Forecasting 1: We first estimate 2 factors $\hat{F}_{t}$ from $X_{t}$ by PCA, which are then used as predictors in $\hat{y}_{t+1 \mid t}=\hat{a}^{\prime} \hat{F}_{t}$, where $\hat{a}$ is the OLS estimate of $a$ in the regression of $y_{t+1}$ on $\hat{F}_{t}$.

Forecasting 2: We first estimate 2 factors $\hat{F}_{t}$ from $X_{t}$ by PCA, and then use $\hat{F}_{t}$ and $\hat{F}_{t} \mathbf{1}(t>\tau)$ as predictors. $\hat{y}_{t+1 \mid t}=\hat{a}^{\prime}\left[\hat{F}_{t}^{\prime} \hat{F}_{t} \mathbf{1}(t>\right.$ $\left.\tau)^{\prime}\right]^{\prime}$, where $\hat{a}$ is the OLS estimate of $a$ in the regression of $y_{t+1}$ on $\hat{F}_{t}$ and $\hat{F}_{t} \mathbf{1}(t>\tau)$.

Forecasting 3: We first estimate 4 factors (replicating $r+k_{1}=$ 4) $\hat{F}_{t}$ from $X_{t}$ by PCA, which are then used as predictors in $\hat{y}_{t+1 \mid t}=$ $\hat{a}^{\prime} \hat{F}_{t}$, where $\hat{a}$ is the OLS estimate of $a$ in the regression of $y_{t+1}$ on $\hat{F}_{t}$.

The above forecasting exercises are implemented recursively, e.g., at each time $t$, the data $X_{t}, X_{t-1}, \ldots, X_{1}$ and $y_{t}, y_{t-1}, \ldots, y_{1}$ are treated as known to forecast $y_{t+1}$. This process starts from $t=149$ to $t=199$, and the mean square errors (MSEs) are calculated by

MSE $=\sum_{t=149}^{199} \frac{\left(y_{t+1}-\hat{y}_{t+1 \mid t}\right)^{2}}{51}$.

To facilitate the comparisons, the MSEs of the Benchmark Forecasting is standardized to be 1 .

The results obtained from 1000 replications are reported in Fig. 1, plotting MSEs against the different break sizes in the abovementioned range. It is clear that the MSEs of the Forecasting 1 method increases drastically with the size of the breaks, in line with our discussion in Section 3. By contrast, the Forecasting 2 and 3 procedures perform equally well and their MSEs remain constant as the break size increases. Notice, however, that they cannot outperform the benchmark forecasting due to the estimation 
Table 1

Empirical sizes of the nominal 5\% size tests for different choices of $\bar{r}$ when $r=3$.

\begin{tabular}{|c|c|c|c|c|c|c|c|c|c|c|c|c|c|}
\hline \multirow[t]{2}{*}{$N$} & \multirow[t]{2}{*}{$T$} & \multicolumn{4}{|c|}{$\hat{\underline{\alpha}}_{0.05} \mid \bar{r}=2$} & \multicolumn{4}{|c|}{$\hat{\alpha}_{0.05} \mid \bar{r}=3$} & \multicolumn{4}{|c|}{$\underline{\hat{\alpha}_{0.05} \mid \bar{r}}=4$} \\
\hline & & LM & Sup LM & Wald & Sup Wald & LM & Sup LM & Wald & Sup Wald & LM & Sup LM & Wald & Sup Wald \\
\hline 100 & 100 & 5.0 & 1.0 & 5.9 & 4.8 & 2.3 & 0.2 & 4.2 & 6.7 & 0.5 & 0.2 & 1.3 & 11.6 \\
\hline 100 & 150 & 5.0 & 1.9 & 4.9 & 3.1 & 3.5 & 0.7 & 3.7 & 4.8 & 1.1 & 0.3 & 1.9 & 7.0 \\
\hline 100 & 200 & 5.7 & 2.7 & 5.0 & 4.0 & 4.9 & 1.8 & 4.0 & 3.5 & 3.0 & 0.5 & 2.9 & 3.9 \\
\hline 100 & 250 & 5.3 & 3.2 & 5.3 & 3.9 & 4.4 & 1.8 & 4.7 & 3.2 & 2.3 & 0.9 & 3.4 & 3.1 \\
\hline 100 & 300 & 6.2 & 4.5 & 6.7 & 4.0 & 5.3 & 2.0 & 5.1 & 3.4 & 3.8 & 1.1 & 4.7 & 3.9 \\
\hline 150 & 100 & 5.3 & 1.2 & 5.9 & 5.1 & 2.6 & 0.2 & 4.0 & 7.9 & 0.8 & 0.2 & 2.3 & 12.9 \\
\hline 150 & 150 & 5.9 & 1.8 & 5.2 & 4.0 & 2.9 & 0.5 & 3.4 & 4.0 & 1.3 & 0.3 & 2.7 & 6.1 \\
\hline 150 & 200 & 5.5 & 2.6 & 6.2 & 4.5 & 3.5 & 1.2 & 5.1 & 3.4 & 2.3 & 0.9 & 3.0 & 4.3 \\
\hline 150 & 250 & 6.0 & 2.9 & 6.9 & 3.8 & 3.5 & 1.6 & 5.7 & 3.1 & 3.2 & 0.5 & 3.6 & 4.7 \\
\hline 150 & 300 & 5.8 & 3.7 & 6.3 & 4.4 & 3.9 & 2.5 & 5.1 & 4.0 & 3.5 & 1.3 & 4.0 & 3.7 \\
\hline 200 & 100 & 4.6 & 1.1 & 5.4 & 5.0 & 2.3 & 0.1 & 3.0 & 8.6 & 0.4 & 0.4 & 1.5 & 15.6 \\
\hline 200 & 150 & 4.7 & 2.3 & 5.6 & 3.2 & 2.8 & 0.2 & 3.7 & 4.3 & 1.2 & 0.1 & 2.7 & 5.6 \\
\hline 200 & 200 & 5.4 & 3.0 & 5.1 & 2.9 & 4.0 & 1.6 & 3.4 & 2.5 & 2.6 & 1.3 & 3.2 & 3.5 \\
\hline 200 & 250 & 6.2 & 3.7 & 7.0 & 4.0 & 3.8 & 2.0 & 6.8 & 4.1 & 2.4 & 1.1 & 4.1 & 5.2 \\
\hline 200 & 300 & 5.3 & 3.1 & 5.5 & 4.6 & 3.2 & 1.5 & 3.5 & 4.0 & 3.4 & 1.3 & 2.6 & 4.5 \\
\hline 250 & 100 & 5.2 & 0.8 & 7.4 & 5.1 & 2.1 & 0.4 & 4.5 & 7.0 & 0.6 & 0.2 & 3.5 & 12.9 \\
\hline 250 & 150 & 4.1 & 2.5 & 5.7 & 3.6 & 2.9 & 0.5 & 3.9 & 4.2 & 1.6 & 0.0 & 2.4 & 6.4 \\
\hline 250 & 200 & 5.3 & 2.6 & 6.5 & 4.9 & 3.5 & 0.8 & 4.6 & 5.0 & 2.9 & 0.3 & 3.4 & 5.2 \\
\hline 250 & 250 & 5.3 & 3.1 & 6.2 & 4.3 & 4.7 & 1.8 & 5.6 & 3.1 & 4.0 & 0.7 & 3.5 & 3.6 \\
\hline 250 & 300 & 5.5 & 4.0 & 5.1 & 3.7 & 4.3 & 1.5 & 4.0 & 3.3 & 3.4 & 1.4 & 2.9 & 3.7 \\
\hline 300 & 100 & 4.7 & 0.6 & 5.2 & 5.4 & 1.5 & 0.2 & 3.4 & 8.5 & 0.3 & 0.3 & 2.9 & 14.0 \\
\hline 300 & 150 & 4.6 & 1.8 & 6.4 & 5.4 & 2.9 & 0.8 & 4.8 & 4.7 & 1.7 & 0.5 & 2.8 & 7.0 \\
\hline 300 & 200 & 3.7 & 2.6 & 7.0 & 4.0 & 3.2 & 0.8 & 6.5 & 4.1 & 1.7 & 0.5 & 4.2 & 5.5 \\
\hline 300 & 250 & 5.9 & 3.5 & 6.3 & 4.1 & 4.8 & 1.7 & 5.2 & 3.4 & 2.7 & 1.0 & 3.3 & 3.5 \\
\hline 300 & 300 & 5.7 & 4.2 & 4.2 & 4.1 & 6.2 & 3.2 & 4.4 & 3.4 & 3.9 & 1.4 & 2.8 & 3.2 \\
\hline 1000 & 1000 & 5.7 & 6.1 & 7.1 & 5.9 & 5.8 & 4.2 & 6.2 & 4.9 & 6.5 & 4.7 & 5.8 & 3.5 \\
\hline
\end{tabular}

Notes: The DGP is $X_{i t}=\sum_{k=1}^{3} \alpha_{i k} F_{k t}+e_{i t}$ where $F_{k t}=\phi_{k} F_{k, t-1}+v_{k t}, \alpha_{i k}, e_{i t}, v_{k t} \sim$ i.i.d. $N(0,1)$, and $\left[\phi_{1}, \phi_{2}, \phi_{3}\right]=[0.8,0.5,0.2]($ see Section 5.2).

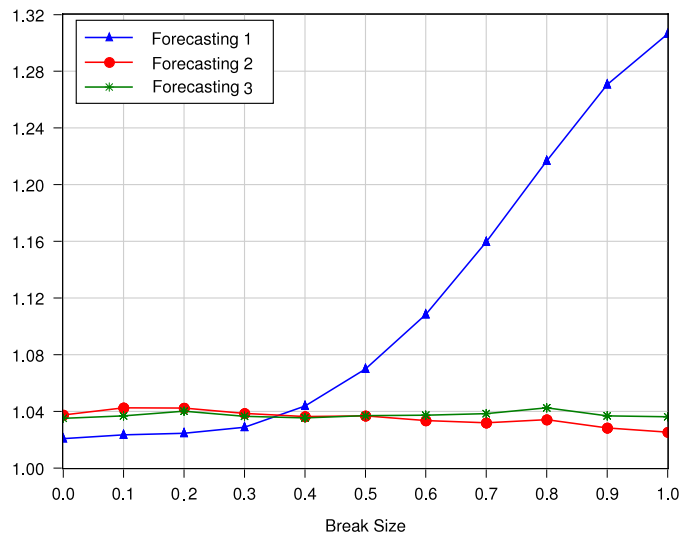

Fig. 1. The MSEs of different forecasting methods in the presence of big breaks (see Section 5.1).

errors of the factors for the chosen sizes of $N$ and $T$. In line with our previous analysis, the lesson to be drawn from this exercise is that, in case of a big break, imposing the number of factors a priori can significantly worsen forecasts.

\subsection{Size properties}

We first simulate data from the following DGP:

$X_{i t}=\sum_{k=1}^{r} \alpha_{i k} F_{k t}+e_{i t}$

where $r=3, \alpha_{i k}$ and $e_{i t}$ are generated as i.i.d standard normal variables, and $\left\{F_{k t}\right\}$ are generated as:

$F_{k t}=\phi_{k} F_{k, t-1}+v_{k t}$

where $\left[\phi_{1}, \phi_{2}, \phi_{3}\right]=[0.8,0.5,0.2]$, and $v_{k t}$ is another i.i.d standard normal error term. The number of replications is 1000 . We consider both the LM and Wald tests and their Sup-type versions.
Table 1 reports the empirical sizes (in percentages) for the LM/Wald tests and Sup-LM/Wald tests using 5\% asymptotic critical values for sample sizes ( $N$ and $T$ ) equal to 100,150,200, 250, 300 and $1000 .{ }^{12}$ We consider three cases regarding the choice of the number of factors to be estimated by PC: (i) the correct one $(\bar{r}=r=3$ ), (ii) smaller than the true number of factors $(\bar{r}=2<$ $r=3$ ), and (iii) larger than the true number of factors $(\bar{r}=4>$ $r=3) .{ }^{13}$ Although, for $N, T \geq 100$, BN's (2002) IC very often select 3 factors, there are some cases where 2 and 4 are also selected.

Broadly speaking, the LM and Wald tests are slightly undersized for $r=2$ and 3, and especially so when $r=4$. Yet, the empirical sizes converge to the nominal size as $N$ and $T$ increase. This finite sample problem is more accurate with the Sup-LM test especially for small $T$, in line with the findings in other studies (see Diebold and Chen, 1996). This is hardly surprising because, for instance, when $T=100$ and $\Pi=[0.15,0.85]$, we only have 15 observations in the first subsample. By contrast, although the Sup-Wald test is too liberal for $T=100$, in general it behaves better than the Sup-LM test (see Kim and Perron, 2009). Theoretically, the asymptotic distribution in Theorem 1 applies only for $\bar{r}=2$, 3. Yet, the results in Table 1 show that this distribution also provides reasonably good approximations for the case $\bar{r}=4$, the only exception being the Sup-Wald test, which is oversized for $T=100$.

To further study how the size of our Wald tests is affected by the selection of $\bar{r}$ in a more general setup, we repeat the above simulations by generating the idiosyncratic errors in the following

\footnotetext{
12 As mentioned earlier, the critical values of the Sup-type tests are taken from Andrews (1993).

13 Notice that the choice of $r=3$ allows us to analyze the consequences of performing our proposed test with the under-parameterized choice of $\bar{r}=2$, where two factors are needed to perform the LM/Wald tests. Had we chosen $r=2$ as the true number of factors, then the test could not be implemented for $\bar{r}=1$.
} 
way as in BN (2002):

$e_{i t}=\rho e_{i, t-1}+u_{i t}+\beta \sum_{h=i-J}^{i-1} u_{h t}+\beta \sum_{h=i+1}^{i+J} u_{h t}$

where $u_{i t} \sim$ i.i.d. $N(0,1), \beta$ and $J$ control the cross sectional correlation of $e_{i t}$, and $\rho$ controls the serial correlation of $e_{i t}$. The other parts of the DGP remain the same as above, and we consider three different types of $e_{t}$ : (1) only serially correlated $(\rho=0.2,0.5,0.7)$, (2) only cross sectionally correlated $(\beta=0.2,0.5, J=5,10)$, (3) both cross sectionally and serially correlated. To save space, we fix $N=100$ (similar to the data set in our application) and focus on the role of $T .^{14}$

The results are reported in Table 2 for the Wald and SupWald tests, where two conclusions can be drawn. First, the serial correlations of $e_{t}$ generally have a larger impact on the sizes than the cross-sectional correlations. Second, the size distortions of the Wald tests disappear as the sample size grows. Overall, the Wald tests have correct sizes for $\bar{r}=4$ when $T=200 .{ }^{15}$

\subsection{Power properties}

We next consider similar DGPs as in Table 1 but this time with $r=2$ and now subject to big breaks which are characterized as deterministic shifts in the means of the factor loadings. ${ }^{16}$ The factors are simulated as $\operatorname{AR}(1)$ processes with coefficients of 0.8 for the first factor and 0.2 for the second. The shifts in the loadings are 0.2 and 0.4 at time $\tau=T / 2$. The other parts of the DGP are the same as in Table 1 . Table 3 reports the empirical powers of the LM/Wald and Sup-LM/Wald tests in percentage terms with 1000 replications. As expected, both tests are powerful to detect the breaks as long as $\bar{r}>r=2$, while the power is trivial when $\bar{r}=r=2$.

Next, we study the powers of our tests when the argument in Section 4.3 fails, i.e., the first element of $\rho_{1}$ and $\rho_{2}$ are both zero. The DGPs are constructed as follows:

$X_{i t}=A_{1} F_{1 t}+A_{2} F_{2 t}+e_{1 t}$ for $t=1, \ldots, T / 2$

$X_{i t}=B_{1} F_{1 t}+A_{2} F_{2 t}+e_{1 t}$ for $t=T / 2+1, \ldots, T$,

where $F_{1 t}$ and $F_{2 t}$ are two $\operatorname{AR}(1)$ process defined as above, $A_{1} \sim$ $N(0,1), A_{2} \sim 0.9 \cdot N(0,1), B_{1} \sim 0.8 \cdot N(0,1)$, and $e_{i t} \sim N(0,1)$. Define $G_{t}^{1}=F_{1 t} \mathbf{1}(t \geq \tau), \Gamma=\left[A_{1} A_{2}\left(B_{1}-A_{1}\right)\right]$, and $F_{t}^{*}=$ $\left(F_{1 t} F_{2 t} G_{t}^{1}\right)^{\prime}$. Then, if $\bar{r}=3$, using results of Bai (2003) it is easy to show that ${ }^{17}$ :

$\hat{F}_{t}=D^{*} F_{t}^{*}+o_{p}(1)$ for $t=1, \ldots, T$,

where

$D^{*}=\left(\begin{array}{ccc}0 & 0.6 & 0 \\ -1.38 & 0 & 1.38 \\ 0 & 0 & -1.38\end{array}\right)$.

It then follows from the definition of $F_{t}^{*}$ that $\rho_{1}$ and $\rho_{2}$ should be vectors taking the form $(0,0, a)$ and $(0, b, 0)$, respectively, with $a, b \neq 0$. Our tests are applied to such DGPs for $\bar{r}=2,3,4$, first

\footnotetext{
14 More simulation results with different $N$ and $T$ are available upon request.

15 The only exception is when the errors are all strongly correlated $(\rho=0.7$ implies that the errors are even more persistent than two of the factors). In practice, this can be tested since $e_{t}$ can be consistently estimated.

16 The results with other types of breaks, such as random shifts, are similar and available upon request.

17 The matrix $D^{*}$ is defined as in Section 4.1 with $\Sigma_{A}$ and $\Sigma_{F}$ replaced by $\Sigma_{\Gamma}$ and $\Sigma_{F^{*}}$.
}

using $\hat{F}_{1 t}$ as the regressand and then $\hat{F}_{2 t}$. The results with 1000 replications are reported in Tables 4 and 5 .

First, it is clear that, even for this special DGP, our Wald and Sup-Wald tests exhibit good power in finite samples when $\hat{F}_{1 t}$ is used as the regressand. Second, although our LM and Sup-LM tests lose power when $\hat{F}_{1 t}$ is the regressand, this is not the case when $\hat{F}_{2 t}$ is used as the regressand. Finally, the Wald and Sup-Wald tests strongly dominates the LM and Sup-LM tests in term of power. Many other simulations have been run in which the power of our Wald and Sup-Wald tests are found to be strong and robust. As will be discussed below, the differences between the power of our LM and Wald tests for this DGP can be explained by the fact that the former uses much less information than the latter. Therefore, we recommend the use of Wald and Sup-Wald on the basis of their good size and power properties.

\subsection{Comparison with the BE test}

As discussed earlier, the $B E$ test relies on the consistent estimation of the original factors. These authors construct $N$ test statistics $s_{i}$ for each of the hypothesis $\alpha_{i}=\beta_{i}$, but not for the joint hypothesis $A=B$. Their method have two limitations. First, as we have shown, the big breaks lead to a new FM representation, as in (5), in which the new factor loadings are constant. Thus, the BE test will lose power when the number of factors is chosen to be $r+k_{1}$, which is quite possible in light of our Proposition 2. On the contrary, our tests will not suffer from this problem when $\bar{r}>r$. To compare the performances of our test and the BE test for the joint hypothesis $(A=B)$, we need to construct the following pooled statistic as suggested by Remark A of BE (2011):

$$
\frac{\sum_{i=1}^{N} s_{i}-N \bar{r}}{\sqrt{2 N \bar{r}}}
$$

where $s_{i}$ is the individual LM statistics in BE (2011). This test should converge to a standard normal distribution as long as $e_{i t}$ and $e_{j t}$ are independent, a restrictive assumption that we also adopt here for comparative purpose. For simplicity, we only report results for the case of known break dates.

We first generate FMs with $r=2$, and compare the performances of the pooled BE test with our Wald test under the null. The DGPs are similar to those used in the size study. The second set of columns in Table 6 (no break) reports the 5\% empirical sizes. In general, we find that both tests exhibit similar sizes.

Then, we generate a break in the loadings of the first factor while the other elements of the DGPs remain the same as in Table 3 where we study the power properties. The break is generated as a shift of size 0.1 in the mean of the loadings. As before, we consider two cases: (i) the number of factors is correctly selected: $\bar{r}=r=$ 2 ; and (ii) the selected number of factors is larger than the true one: $\bar{r}=3>r=2$. The third and fourth columns in Table 6 report the empirical rejection rates of both tests. In agreement with our previous discussion, the differences in power are quite striking: when $\bar{r}=2$, the pooled BE test is much more powerful while the opposite occurs when $\bar{r}=3$. Notice that, as discussed above, for $\bar{r}=2$, our test will not be able to detect the break whereas, for $\bar{r}=3$, the pooled BE test will be powerless. However, according to our Proposition 2, the use of BN's (2002) IC will yield the choice of $\bar{r}=3$ as a much more likely outcome as $N$ and $T$ increase. For example, for this simulation, on average the $P C_{p 1}$ of $B N(2002)$ chooses $\widehat{r}=3$ in $94.6 \%$ of the cases.

The second problem of the BE approach is that, even when $r$ is assumed to be known, their method may falsely reject the null hypothesis $\alpha_{i}=\beta_{i}$ for some $i$. To see this, we can use the same argument as in (11)-(13) to show that, in the presence of big 


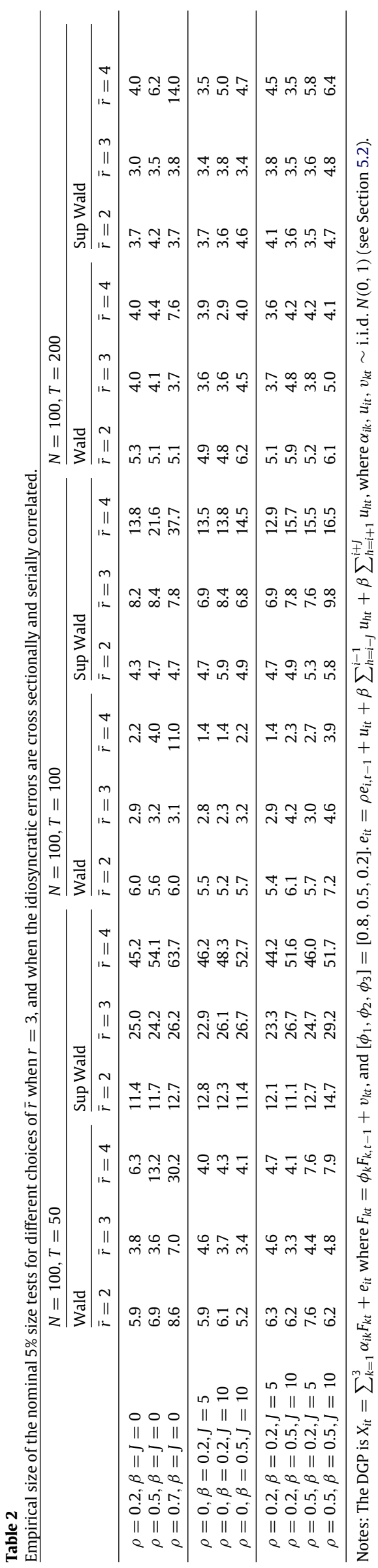


Table 3

Empirical power of nominal 5\% size tests for different choices of $\bar{r}$ when $r=2$ and $k_{1}=2$.

\begin{tabular}{|c|c|c|c|c|c|c|c|c|c|c|c|c|c|}
\hline \multirow[t]{2}{*}{$N$} & \multirow[t]{2}{*}{$T$} & \multicolumn{4}{|c|}{$\hat{\alpha}_{0.05} \mid \bar{r}=2$} & \multicolumn{4}{|c|}{$\hat{\alpha}_{0.05} \mid \bar{r}=3$} & \multicolumn{4}{|c|}{$\hat{\alpha}_{0.05} \mid \bar{r}=4$} \\
\hline & & LM & Sup LM & Wald & Sup Wald & LM & Sup LM & Wald & Sup Wald & LM & Sup LM & Wald & Sup Wald \\
\hline 100 & 100 & 6.3 & 1.8 & 8.1 & 5.4 & 77.9 & 1.8 & 100 & 98.3 & 41.7 & 0.5 & 100 & 97.3 \\
\hline 100 & 150 & 8.9 & 2.5 & 10.0 & 4.8 & 95.8 & 24.0 & 100 & 100 & 88.8 & 2.8 & 100 & 99.9 \\
\hline 100 & 200 & 8.9 & 4.1 & 9.3 & 5.4 & 97.6 & 72.9 & 92.0 & 92.0 & 95.5 & 39.6 & 91.8 & 92.5 \\
\hline 100 & 250 & 12.0 & 5.3 & 12.4 & 6.5 & 99.1 & 98.0 & 97.4 & 97.4 & 99.0 & 77.9 & 97.4 & 97.4 \\
\hline 100 & 300 & 13.0 & 6.5 & 11.6 & 6.0 & 99.6 & 98.0 & 83.6 & 83.6 & 99.4 & 94.1 & 83.5 & 83.7 \\
\hline 150 & 100 & 6.1 & 2.2 & 7.8 & 5.9 & 77.9 & 1.4 & 99.7 & 99.5 & 41.6 & 0.6 & 99.8 & 99.0 \\
\hline 150 & 150 & 7.5 & 2.2 & 8.3 & 5.0 & 95.4 & 24.5 & 100 & 100 & 88.5 & 2.2 & 100 & 100 \\
\hline 150 & 200 & 8.8 & 4.1 & 9.8 & 5.4 & 98.8 & 76.5 & 100 & 100 & 97.7 & 40.2 & 100 & 100 \\
\hline 150 & 250 & 9.7 & 4.8 & 10.3 & 6.0 & 99.4 & 94.4 & 99.0 & 99.1 & 98.5 & 79.1 & 99.0 & 99.1 \\
\hline 150 & 300 & 11.4 & 6.3 & 10.8 & 7.1 & 99.7 & 98.6 & 90.5 & 91.1 & 99.7 & 94.5 & 90.7 & 91.1 \\
\hline 200 & 100 & 6.4 & 1.5 & 7.6 & 4.6 & 79.4 & 2.3 & 100 & 97.7 & 42.9 & 0.7 & 100 & 99.2 \\
\hline 200 & 150 & 8.5 & 3.4 & 9.5 & 6.3 & 97.0 & 24.1 & 100 & 100 & 89.0 & 3.0 & 100 & 100 \\
\hline 200 & 200 & 8.6 & 3.5 & 9.3 & 4.5 & 99.0 & 77.6 & 100 & 100 & 98.0 & 38.8 & 100 & 100 \\
\hline 200 & 250 & 11.5 & 4.5 & 12.3 & 5.7 & 100 & 96.8 & 100 & 100 & 100 & 82.7 & 100 & 100 \\
\hline 200 & 300 & 11.2 & 5.4 & 12.6 & 6.4 & 99.8 & 98.8 & 99.9 & 99.9 & 99.7 & 95.1 & 99.9 & 99.9 \\
\hline 250 & 100 & 5.1 & 1.4 & 6.7 & 4.5 & 80.4 & 1.8 & 100 & 99.7 & 45.2 & 1.0 & 100 & 99.2 \\
\hline 250 & 150 & 6.7 & 2.4 & 7.8 & 5.0 & 97.0 & 24.5 & 99.9 & 100 & 90.7 & 3.2 & 100 & 100 \\
\hline 250 & 200 & 7.2 & 3.4 & 7.8 & 5.0 & 99.2 & 78.9 & 100 & 100 & 98.4 & 40.9 & 100 & 100 \\
\hline 250 & 250 & 10.5 & 5.5 & 11.3 & 5.8 & 99.8 & 95.6 & 100 & 100 & 99.7 & 82.4 & 100 & 100 \\
\hline 250 & 300 & 11.5 & 5.7 & 12.0 & 7.6 & 99.9 & 99.2 & 100 & 100 & 99.9 & 95.1 & 100 & 100 \\
\hline 300 & 100 & 6.0 & 1.6 & 7.0 & 6.7 & 80.1 & 1.2 & 100 & 99.1 & 45.4 & 0.3 & 100 & 98.9 \\
\hline 300 & 150 & 8.6 & 2.1 & 9.9 & 4.7 & 97.3 & 24.9 & 100 & 100 & 91.5 & 3.4 & 100 & 100 \\
\hline 300 & 200 & 8.6 & 4.3 & 9.2 & 6.8 & 99.3 & 79.0 & 100 & 100 & 98.4 & 43.3 & 100 & 100 \\
\hline 300 & 250 & 11.4 & 4.4 & 11.9 & 5.8 & 99.8 & 94.3 & 100 & 100 & 99.5 & 82.6 & 100 & 100 \\
\hline 300 & 300 & 11.3 & 5.9 & 12.1 & 7.7 & 99.8 & 99.0 & 100 & 100 & 99.8 & 96.3 & 100 & 100 \\
\hline
\end{tabular}

Notes: The DGP is $X_{i t}=\sum_{k=1}^{2} \alpha_{i k} F_{k t}+e_{i t}$ where $F_{k t}=\phi_{k} F_{k, t-1}+v_{k t}, \alpha_{i k}, e_{i t}, v_{k t} \sim$ i.i.d. $N(0,1)$, and $\left[\phi_{1}, \phi_{2}\right]=[0.8,0.2]$. The shifts in the means of the factor loadings are 0.4 and 0.2 at $\tau=T / 2$ (see Section 5.3).

Table 4

Empirical power of nominal 5\% size tests for different choices of $\bar{r}$ when $r=2, k_{1}=1$, and $\hat{F}_{1 t}$ is used as the regressand.

\begin{tabular}{|c|c|c|c|c|c|c|c|c|c|c|c|c|c|}
\hline \multirow[t]{2}{*}{$N$} & \multirow[t]{2}{*}{$T$} & \multicolumn{4}{|c|}{$\bar{r}=2$} & \multicolumn{4}{|c|}{$\bar{r}=3$} & \multicolumn{4}{|c|}{$\bar{r}=4$} \\
\hline & & LM & Sup LM & Wald & Sup Wald & LM & Sup LM & Wald & Sup Wald & LM & Sup LM & Wald & Sup Wald \\
\hline 50 & 50 & 9.7 & 4.5 & 64.1 & 66.5 & 2.0 & 3.7 & 99.0 & 99.9 & 1.4 & 3.5 & 98.7 & 100 \\
\hline 50 & 100 & 13.9 & 4.3 & 60.5 & 64.0 & 8.7 & 1.4 & 98.3 & 99.5 & 2.0 & 0.7 & 98.2 & 99.7 \\
\hline 50 & 150 & 15.3 & 7.8 & 56.9 & 62.1 & 15.2 & 4.6 & 98.4 & 99.7 & 8.8 & 1.2 & 98.4 & 99.8 \\
\hline 50 & 200 & 17.6 & 11.9 & 56.5 & 62.4 & 19.5 & 9.4 & 97.2 & 100 & 12.3 & 3.4 & 97.6 & 100 \\
\hline 100 & 50 & 10.6 & 4.1 & 69.6 & 72.3 & 3.0 & 4.6 & 99.5 & 100 & 2.3 & 3.7 & 99.5 & 100 \\
\hline 100 & 100 & 14.2 & 5.1 & 67.1 & 70.3 & 9.0 & 2.1 & 99.8 & 100 & 3.5 & 0.9 & 99.8 & 100 \\
\hline 100 & 150 & 15.4 & 10.3 & 63.1 & 68.4 & 13.5 & 4.0 & 99.4 & 100 & 8.0 & 1.2 & 99.2 & 100 \\
\hline 100 & 200 & 17.2 & 11.3 & 67.5 & 72.1 & 16.5 & 7.7 & 99.7 & 100 & 10.5 & 2.2 & 99.7 & 100 \\
\hline 150 & 50 & 9.7 & 5.3 & 73.9 & 76.8 & 2.9 & 3.9 & 99.9 & 100 & 1.2 & 2.9 & 100 & 100 \\
\hline 150 & 100 & 12.2 & 5.3 & 70.5 & 74.1 & 7.8 & 2.1 & 99.8 & 100 & 1.8 & 0.7 & 99.8 & 100 \\
\hline 150 & 150 & 12.2 & 7.3 & 69.6 & 74.7 & 9.3 & 3.0 & 99.7 & 100 & 5.2 & 0.9 & 99.9 & 100 \\
\hline 150 & 200 & 12.3 & 10.7 & 64.9 & 70.5 & 11.7 & 7.6 & 99.9 & 100 & 6.6 & 2.2 & 99.9 & 100 \\
\hline 200 & 50 & 9.4 & 5.8 & 75.3 & 77.3 & 2.9 & 4.3 & 100 & 100 & 1.9 & 3.5 & 100 & 100 \\
\hline 200 & 100 & 10.8 & 5.7 & 71.6 & 76.4 & 8.0 & 2.1 & 99.8 & 100 & 2.6 & 0.8 & 99.9 & 100 \\
\hline 200 & 150 & 13.1 & 10.4 & 70.9 & 79.0 & 9.4 & 4.7 & 99.8 & 100 & 5.6 & 0.9 & 99.9 & 100 \\
\hline 200 & 200 & 13.8 & 10.2 & 73.1 & 76.8 & 13.7 & 7.4 & 100 & 100 & 8.4 & 2.0 & 100 & 100 \\
\hline
\end{tabular}

Notes: The DGP is $X_{i t}=\alpha_{i 1} F_{1 t}+\alpha_{i 2} F_{2 t}+e_{i t}$ for $t=1, \ldots, T / 2$, and $X_{i t}=\alpha_{i 1} F_{1 t}+\beta_{i 2} F_{2 t}+e_{i t}$ for $t=T / 2+1, \ldots, T$, where $F_{k t}=\phi_{k} F_{k, t-1}+v_{k t}, \alpha_{i 1}, \alpha_{i 2}, \beta_{i 2}, e_{i t}, v_{k t} \sim$ i.i.dN $(0,1)$, and $\left[\phi_{1}, \phi_{2}\right]=[0.8,0.2]$ (see Section 5.3).

breaks, for some variables with constant loadings, ${ }^{18}$ replacing the true factors by the estimated factors will result in a "break" in the factor loadings.

\subsection{Comparison with the HI test}

The HI (2012) test is based on the comparison of the covariance matrices of the estimated factors before and after the break. In view

\footnotetext{
18 This is possible since our definition for big breaks allows a fixed proportion of variables to have no breaks.
}

of our results in (9) and (10), $\tau^{-1} \sum_{t=1}^{\tau} \hat{F}_{t} \hat{F}_{t}^{\prime}=D_{1} \hat{\Sigma}_{F}^{1} D_{1}^{\prime}+o_{p}(1)$, and $(T-\tau)^{-1} \sum_{t=\tau+1}^{T} \hat{F}_{t} \hat{F}_{t}^{\prime}=D_{2}^{\dagger} \hat{\Sigma}_{F}^{2} D_{2}^{\dagger^{\prime}}+o_{p}(1)$, where $\hat{\Sigma}_{F}^{1}=$ $\tau^{-1} \sum_{t=1}^{\tau} F_{t} F_{t}^{\prime}$ and $\hat{\Sigma}_{F}^{2}=(T-\tau)^{-1} \sum_{t=\tau+1}^{T} F_{t} F_{t}^{\prime}$. Therefore, the HI test is able to detect breaks if $\hat{\Sigma}_{F}^{1}, \hat{\Sigma}_{F}^{2} \rightarrow \Sigma_{F}$ and $D_{1}$ and $D_{2}^{\dagger}$ converge to different limits as $N$ and $T$ go to infinity. Specifically, their test is defined as:

$T\left(\mathcal{C}(\pi)^{\prime} \hat{V}^{-1} \mathcal{C}(\pi)\right)$

where

$\mathcal{C}(\pi)=\operatorname{vech}\left[\frac{1}{\tau} \sum_{1}^{\tau} \hat{F}_{t} \hat{F}_{t}^{\prime}-\frac{1}{T-\tau} \sum_{\tau+1}^{T} \hat{F}_{t} \hat{F}_{t}^{\prime}\right]$, 
Table 5

Empirical power of nominal 5\% size tests for different choices of $\bar{r}$ when $r=2, k_{1}=1$, and $\hat{F}_{2 t}$ is used as the regressand.

\begin{tabular}{|c|c|c|c|c|c|c|c|c|c|c|c|c|c|}
\hline \multirow[t]{2}{*}{$N$} & \multirow[t]{2}{*}{$T$} & \multicolumn{4}{|c|}{$\bar{r}=2$} & \multicolumn{4}{|c|}{$\bar{r}=3$} & \multicolumn{4}{|c|}{$\bar{r}=4$} \\
\hline & & LM & Sup LM & Wald & Sup Wald & LM & Sup LM & Wald & Sup Wald & LM & Sup LM & Wald & Sup Wald \\
\hline 50 & 50 & 9.7 & 4.5 & 20.2 & 32.1 & 17.8 & 2.5 & 94.4 & 80.6 & 3.0 & 2.7 & 87.1 & 80.2 \\
\hline 50 & 100 & 13.9 & 4.3 & 16.6 .5 & 23.3 & 87.8 & 2.1 & 98.9 & 88.9 & 78.7 & 0.3 & 98.6 & 84.7 \\
\hline 50 & 150 & 15.3 & 7.8 & 18.0 & 20.9 & 92.5 & 78.4 & 99.7 & 100 & 89.9 & 21.9 & 99.5 & 97.0 \\
\hline 50 & 200 & 17.6 & 11.9 & 19.5 & 22.3 & 95.1 & 90.9 & 99.7 & 100 & 93.0 & 85.9 & 99.7 & 100 \\
\hline 100 & 50 & 10.6 & 6.1 & 19.5 & 33.7 & 18.8 & 2.8 & 96.0 & 84.8 & 2.6 & 3.3 & 91.6 & 83.8 \\
\hline 100 & 100 & 14.2 & 5.1 & 19.5 & 25.1 & 87.4 & 1.9 & 99.6 & 90.6 & 79.9 & 1.4 & 99.5 & 87.1 \\
\hline 100 & 150 & 15.4 & 10.3 & 18.3 & 21.1 & 93.8 & 81.6 & 99.4 & 100 & 90.9 & 25.3 & 99.2 & 97.1 \\
\hline 100 & 200 & 17.2 & 11.3 & 19.2 & 20.6 & 94.7 & 90.9 & 99.6 & 100 & 93.3 & 85.2 & 99.5 & 100 \\
\hline 150 & 50 & 9.7 & 5.3 & 17.4 & 32.2 & 20.8 & 2.9 & 97.1 & 87.9 & 3.1 & 2.8 & 93.0 & 86.4 \\
\hline 150 & 100 & 12.2 & 5.3 & 17.1 & 23.0 & 88.4 & 2.1 & 99.9 & 93.3 & 80.3 & 0.8 & 99.9 & 90.6 \\
\hline 150 & 150 & 12.2 & 7.3 & 14.3 & 23.3 & 93.3 & 79.1 & 99.7 & 100 & 91.2 & 23.0 & 99.6 & 98.1 \\
\hline 150 & 200 & 12.3 & 10.7 & 14.7 & 19.3 & 94.5 & 90.7 & 99.7 & 100 & 93.6 & 85.7 & 99.7 & 100 \\
\hline 200 & 50 & 9.4 & 5.8 & 17.7 & 32.7 & 20.0 & 3.9 & 96.5 & 87.8 & 2.4 & 4.7 & 92.4 & 87.0 \\
\hline 200 & 100 & 10.8 & 5.7 & 15.1 & 24.1 & 87.4 & 2.4 & 100. & 94.4 & 78.4 & 1.1 & 99.9 & 90.9 \\
\hline 200 & 150 & 13.1 & 10.4 & 15.3 & 23.3 & 93.1 & 80.7 & 99.9 & 100 & 91.2 & 26.2 & 99.8 & 98.4 \\
\hline 200 & 200 & 13.8 & 10.2 & 15.8 & 20.3 & 93.9 & 89.6 & 99.7 & 100 & 92.3 & 84.9 & 99.7 & 100 \\
\hline
\end{tabular}

Notes: DGP of Table 4 (see Section 5.3).

and $\hat{V}$ is a HAC estimator of the covariance matrix of $\mathcal{C}(\pi)$ which is either constructed using the whole sample (LM version of the test) or using subsamples before and after the break (Wald version).

Basically, the HI test exploits the same insight as our tests in converting an infinite-dimensional problem to a finite one, except that it relies on a different use of the estimated factors. Compared to ours, the HI test uses more information since our LM test only uses the first row (except the first element) of $\frac{1}{\tau} \sum_{t=1}^{\tau} \hat{F}_{t} \hat{F}_{t}^{\prime}$, while our Wald test uses all the elements of the matrix except the first one $\left(\frac{1}{\tau} \sum_{1}^{\tau} \hat{F}_{1 t}^{2}\right)$.

In principle, it may seem that the use of less information is the price one has to pay to render our testing procedure much simpler than theirs. After all, both steps in our approach can be easily implemented in any conventional statistical software used by practitioners, while HI's test is computationally more burdensome. Yet, our Wald test exhibits very similar power to theirs in all the simulations we have run. HI (2012) reports some simulation results for the power comparisons with our Wald tests under very general DGPs. Therefore, to avoid repetitions, we focus only on small samples $(N, T \leq 100)$, and compare the (size-adjusted) power curves of HI's and our Wald tests (using the Bartlett kernel) for the following DGP: $X_{i t}=A_{1 i} F_{t}+e_{i t}$ for $t=1, \ldots, T / 2$, and $X_{i t}=\left(A_{1 i}+b\right) F_{t}+e_{i t}$ for $t=T / 2+1, \ldots, T$, where $F_{t}=\rho F_{t-1}+u_{t}$, $A_{1 i}, u_{t}, e_{i t} \sim$ i.i.d $N(0,1), \rho=0.8$, and $b$ is the break size which ranges from 0 to 0.5 . As can be observed in Fig. 2, our Wald test has better power properties than HI's test in all these cases. However, not surprisingly, as $N$ and $T$ get large, both tests perform very similarly in term of power. ${ }^{19}$

\section{An empirical application}

To provide an empirical application of our tests, we use Stock and Watson's (2009) data set consisting of 144 quarterly time series of nominal and real variables for the US ranging from 1959:Q1 to 2006:Q4. Since not all the variables are available for the whole period, we end up using their suggested balanced panel of standardized variables with $T=190, N=109$. This more or less corresponds to the case where $T=200, N=100$ in Tables 1 and 2, where no severe size distortions are found. We refer to Stock

\footnotetext{
19 This can be seen from Tables 5A and 5B of HI (2012). We also have similar unreported simulation results that are available upon request.
}

Table 6

Size and power comparisons of BE (2011) and our Wald test at nominal 5\% size for $r=2$.

\begin{tabular}{|c|c|c|c|c|c|c|c|}
\hline \multirow[t]{2}{*}{$N$} & \multirow[t]{2}{*}{$T$} & \multicolumn{2}{|c|}{ no break, $\bar{r}=2$} & \multicolumn{2}{|c|}{1 break, $\bar{r}=2$} & \multicolumn{2}{|c|}{1 break, $\bar{r}=3$} \\
\hline & & $\mathrm{BE}$ & Wald & $\mathrm{BE}$ & Wald & $\mathrm{BE}$ & Wald \\
\hline 100 & 100 & 6.0 & 3.9 & 100 & 5.6 & 21.9 & 96.8 \\
\hline 100 & 150 & 5.9 & 5.2 & 100 & 7.2 & 18.2 & 100 \\
\hline 100 & 200 & 5.2 & 4.3 & 100 & 6.2 & 26.0 & 89.8 \\
\hline 100 & 250 & 5.3 & 4.8 & 100 & 8.7 & 17.9 & 97.7 \\
\hline 100 & 300 & 5.7 & 4.3 & 100 & 7.4 & 30.2 & 83.9 \\
\hline 150 & 100 & 6.4 & 4.3 & 100 & 5.8 & 18.3 & 94.6 \\
\hline 150 & 150 & 5.9 & 5.7 & 100 & 6.6 & 16.2 & 100 \\
\hline 150 & 200 & 5.6 & 4.3 & 100 & 6.2 & 12.5 & 100 \\
\hline 150 & 250 & 5.5 & 4.5 & 100 & 5.7 & 14.9 & 98.3 \\
\hline 150 & 300 & 4.9 & 4.0 & 100 & 5.6 & 20.6 & 89.7 \\
\hline 200 & 100 & 5.5 & 4.1 & 100 & 4.1 & 20.0 & 95.8 \\
\hline 200 & 150 & 5.4 & 4.8 & 100 & 6.6 & 15.8 & 100 \\
\hline 200 & 200 & 7.0 & 4.5 & 100 & 6.3 & 14.0 & 100 \\
\hline 200 & 250 & 6.5 & 4.7 & 100 & 7.5 & 12.6 & 100 \\
\hline 200 & 300 & 5.0 & 4.7 & 100 & 7.8 & 12.0 & 99.7 \\
\hline 250 & 100 & 6.8 & 3.9 & 100 & 4.2 & 18.8 & 97.0 \\
\hline 250 & 150 & 5.4 & 5.3 & 100 & 5.9 & 14.9 & 100 \\
\hline 250 & 200 & 4.5 & 4.6 & 100 & 6.1 & 11.3 & 100 \\
\hline 250 & 250 & 5.1 & 4.2 & 100 & 6.6 & 10.9 & 100 \\
\hline 250 & 300 & 6.6 & 4.9 & 100 & 8.3 & 7.9 & 100 \\
\hline 300 & 100 & 7.3 & 4.7 & 100 & 5.4 & 19.7 & 96.3 \\
\hline 300 & 150 & 7.0 & 3.6 & 100 & 6.1 & 14.4 & 100 \\
\hline 300 & 200 & 5.9 & 3.4 & 100 & 6.0 & 13.6 & 100 \\
\hline 300 & 250 & 5.9 & 5.4 & 100 & 6.7 & 12.0 & 100 \\
\hline 300 & 300 & 5.7 & 6.1 & 100 & 7.0 & 10.0 & 100 \\
\hline
\end{tabular}

Notes: DGP of Table 3. The shift in the mean of the factor loadings is either zero (no break) or 0.1 (break) (see Section 5.4).

and Watson (2009) for the details of the data description and the standardization procedure.

Using various BN's (2002) IC $\left(I C_{p 1}, I C_{p 2}, P C_{p 1}\right.$ and $\left.P C_{p 2}\right)$ the estimated number of factors ranges from 2 to 6 , therefore we implement our test for $\bar{r}=2-6$. The Sup-Wald test is applied since no priori break date is assumed to be known. In order to have enough observations in both subsamples, we use the trimming $\Pi=[0.3,0.7]$. It corresponds to the time period ranging from 1973:Q3 to 1992:Q3 which includes several relevant events like, e.g., the oil price shocks $(1973,1979)$ and the beginning of great moderation period in the early 1980s. The graphs displayed in Fig. 3 are the series of Wald tests for different values of $\bar{r}$, with the horizontal lines representing the $5 \%$ asymptotic critical values of the Sup-Wald tests.

We find that the Sup-Wald test rejects the null when $\bar{r}=$ 5,6 . The estimated break date is seems to be around 1979 or 

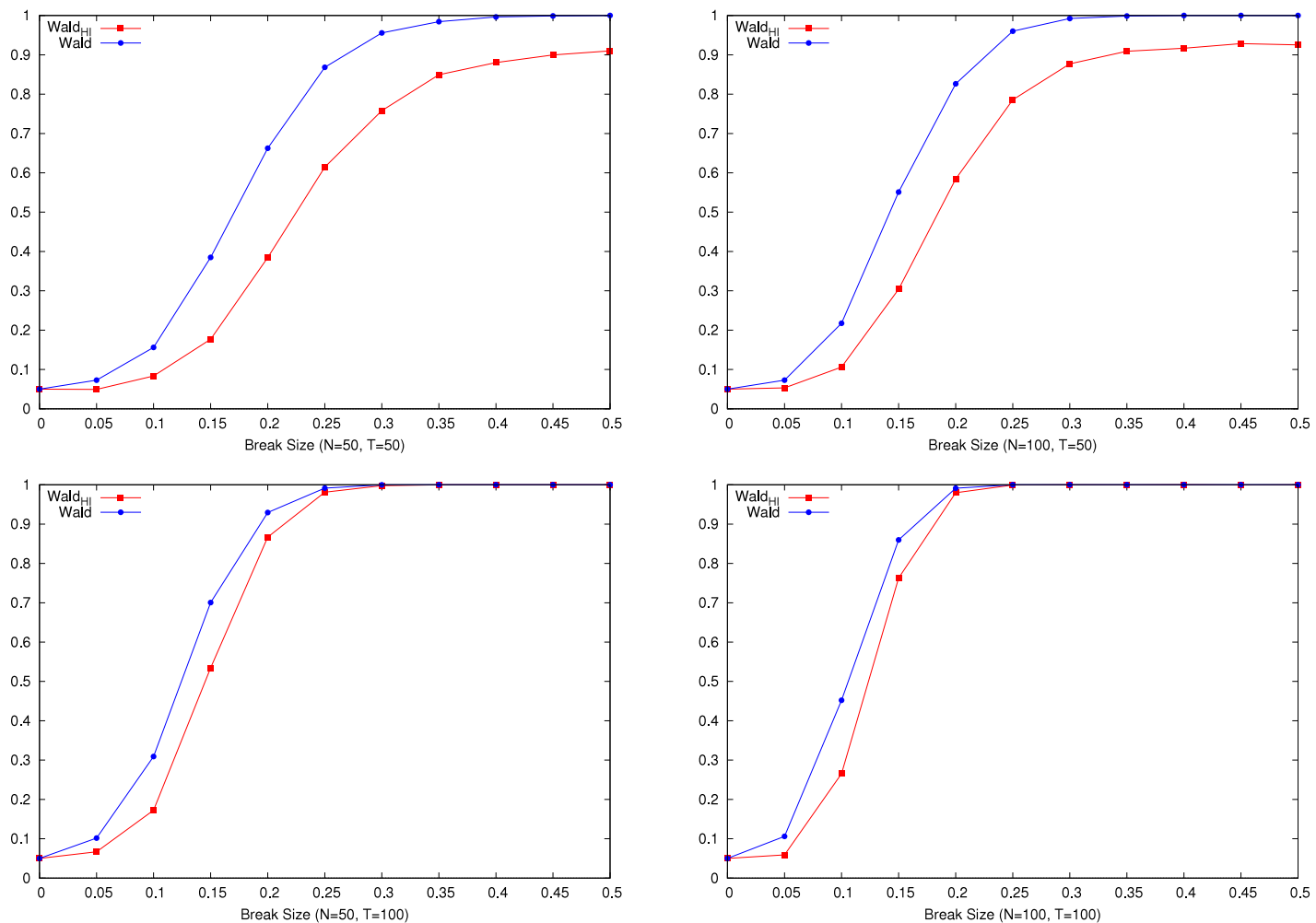

Fig. 2. Power comparison of HI (2012) and our Wald Tests for $N, T \leq 100$ when $r=1$. Notes: The DGP is $X_{i t}=A_{1 i} F_{t}+e_{i t}$ for $t=1, \ldots X_{i t}=\left(A_{1 i}+b\right) F_{t}+e_{i t}$ for $t=T / 2+1, \ldots, T$, where $F_{t}=\phi F_{t-1}+u_{t}, A_{1 i}, u_{t}, e_{i t} \sim$ i.i.d $N(0,1)$, and $\phi=0.8$. The reported curves are the size adjusted power curves of our Wald test (blue) and HI's Wald test (red) when the break size $b$ increases from 0 to 0.5 (see Section 5.5).

1980 (second oil price shock), rather than 1984, which is the only candidate considered by Stock and Watson (2009) as a potential break date in their empirical application with the same data set. One possible explanation for this break date could be the Iranian revolution at the beginning of 1979 and its subsequent impact on monetary policy in the US (see Fernández-Villaverde et al. (2010)).

\section{Conclusions}

In this paper, we propose a simple two-step procedure to test for big structural breaks in the factor loadings of large FM that circumvents some limitations affecting other available tests. In particular, after choosing the number of factors in the whole sample according to BN's (2002) IC and estimating them by PCA, our test relies on a regression of one of the estimated factors on the remaining ones, allowing for a break in the parameters at known or unknown date. LM and Wald tests for the null of parameter stability are applied. We show that our test may have better power than the test of BE (2011) under the alternative of big breaks and, and that it is simpler than the test of HI (2012). Despite using less information than the latter, we show that it is used in a more efficient way, and that our Wald test has better power properties than the HI test when dealing with small samples.

Our testing approach can be easily implemented in any statistical package and it is useful to avoid serious forecasting/estimation problems in standard econometric practices with factors. This could be the case of FAR and FAVAR models, when the factor loadings are subject to big breaks and the number of factors is a priori determined (as is conventional in several macroeconomic and financial applications).

In the second step of our testing approach, a Sup-type test is used to detect a break of the parameters in that regression when the break date is assumed to be unknown. As the simulations show, this test performs very well especially when $T \geq 100$. For smaller samples, as it happens with many other Sup-type tests, bootstrap can improve the finite-sample performance of the test compared to the tabulated asymptotic critical values of Andrews (1993), as suggested by Diebold and Chen (1996). It is high in our research agenda to explore this possibility.

Moreover, as discussed earlier in Remarks 8 and 9, many other existing methods for testing structural changes in linear regressions can also be applied in our second-stage procedure. Further, our testing approach can allow for multiple big breaks through sequential estimation, like in Bai and Perron $(1998,2003)$, for locating the candidate break dates. Exploring further this issue remains also high in our research agenda.

Finally, though a simple testing procedure has been outlined in Section 4.4 to identify whether breaks stem from loadings or from the volatility of the factors, we plan to derive other alternative tests based on the rank of the covariance matrix of the estimated factors in different subsamples which can also be extended to test for other sources of parameter instability.

\section{Appendix}

\section{A.1. Proof of Propositions 1 and 2}

The proof proceeds by showing that the errors, factors and loadings in model (5) satisfy Assumption A-D of BN (2002). Then, once these results are proven, Propositions 1 and 2 just follow immediately from application of Theorems 1 and 2 of BN (2002). Define $F_{t}^{*}=\left[\begin{array}{ll}F_{t}^{\prime} & G_{t}^{1^{\prime}}\end{array}\right]^{\prime}, \epsilon_{t}=H G_{t}^{2}+e_{t}$, and $\Gamma=\left[\begin{array}{ll}A & \Lambda\end{array}\right]$. The proofs of Lemmas 1-8 are similar to those in BN (2002). To save space and avoid repetition, we put them in our online appendix (http://www.eco.uc3m.es/ jgonzalo/WP1.html).

Lemma 1. $E\left\|F_{t}^{*}\right\|^{4}<\infty$ and $T^{-1} \sum_{t=1}^{T} F_{t}^{*} F_{t}^{*^{\prime}} \stackrel{p}{\rightarrow} \Sigma_{F}^{*}$ as $T \rightarrow \infty$ for some positive matrix $\Sigma_{F}^{*}$. 

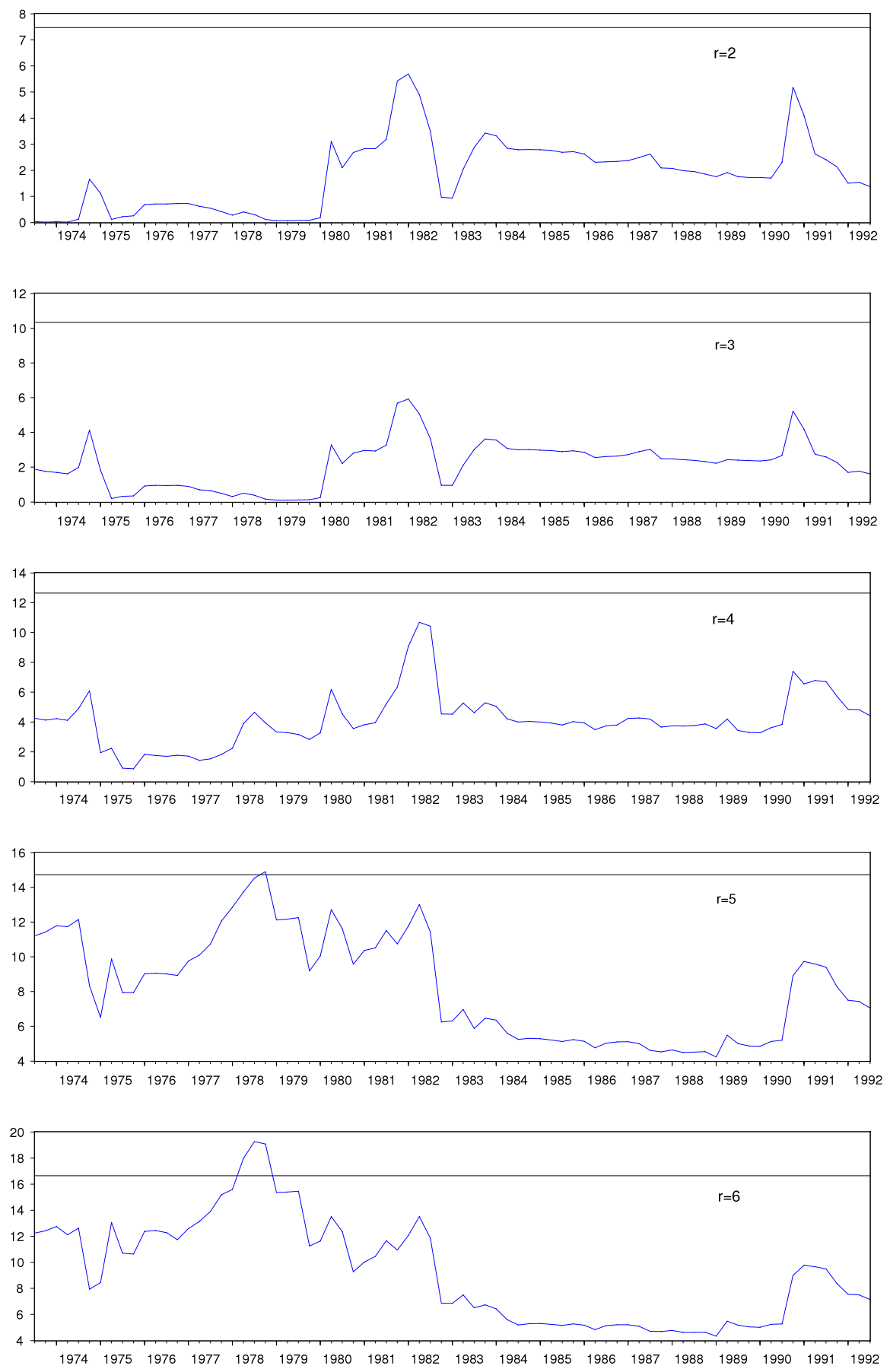

Fig. 3. US data set of Stock and Watson (2009), from 1959:Q1 to 2006:Q4. The trimming $\Pi=[0.3$, 0.7] is used for the Wald tests with $\bar{r}=2$ to 6 (from top to bottom), and the horizontal lines are the corresponding $5 \%$ asymptotic critical values for the Sup-Wald Test.

Lemma 2. $\left\|\Gamma_{i}\right\|<\infty$ for all $i$, and $N^{-1} \Gamma^{\prime} \Gamma \rightarrow \Sigma_{\Gamma}$ as $N \rightarrow \infty$ for some positive definite matrix $\Sigma_{\Gamma}$.

The following lemmas involve the new errors $\epsilon_{t}$. Let $M$ and $M^{*}$ denote some positive constants.

Lemma 3. $E\left(\epsilon_{i t}\right)=0, E\left|\epsilon_{i t}\right|^{8} \leq M^{*}$.

Lemma 4. $E\left(\epsilon_{s}^{\prime} \epsilon_{t} / N\right)=E\left(N^{-1} \sum_{i=1}^{N} \epsilon_{i s} \epsilon_{i t}\right)=\gamma_{N}^{*}(s, t),\left|\gamma_{N}^{*}(s, s)\right|$ $\leq M^{*}$ for all $s$, and $\sum_{s=1}^{T} \gamma_{N}^{*}(s, t)^{2} \leq M^{*}$ for all $t$ and $T$.

Lemma 5. $E\left(\epsilon_{i t} \epsilon_{j t}\right)=\tau_{i j, t}^{*}$ with $\left|\tau_{i j, t}^{*}\right| \leq\left|\tau_{i j}^{*}\right|$ for some $\tau_{i j}^{*}$ and for all $t ;$ and $N^{-1} \sum_{i=1}^{N} \sum_{j=1}^{N}\left|\tau_{i j}^{*}\right| \leq M^{*}$.
Lemma 6. $E\left(\epsilon_{i t} \epsilon_{j s}\right)=\tau_{i j, t s}^{*}$ and $(N T)^{-1} \sum_{i=1}^{N} \sum_{j=1}^{N} \sum_{t=1}^{T} \sum_{s=1}^{T}$ $\left|\tau_{i j, t s}^{*}\right| \leq M^{*}$.

Lemma 7. For every $(t, s), E\left|N^{-1 / 2} \sum_{i=1}^{N}\left[\epsilon_{i s} \epsilon_{i t}-E\left(\epsilon_{i s} \epsilon_{i t}\right)\right]\right|^{4} \leq M^{*}$.

Lemma 8. $E\left(\frac{1}{N} \sum_{i=1}^{N}\left\|\frac{1}{\sqrt{T}} \sum_{t=1}^{T} F_{t}^{*} \epsilon_{i t}\right\|^{2}\right) \leq M^{*}$.

Finally, it is easy to verify that $\sum_{i=1}^{N} \sum_{t=1}^{T}\left\|\eta_{i}\right\|^{2} E\left\|F_{t}\right\|^{2}=O(1)$ and thus the new idiosyncratic errors $\epsilon_{t}$ satisfy the necessary condition for the consistency of $\hat{r}$ (see Observation 1 of Bates et al., 2013).

Once it has been shown that the new factors: $F_{t}^{*}$, the new loadings: $\Gamma$ and the new errors: $\epsilon_{t}$ all satisfy the conditions of 
BN (2002), Propositions 1 and 2 just follow directly from their Theorem 1 and 2, with $r$ replaced by $r+k_{1}$ and $F_{t}$ replaced by $F_{t}^{*}$.

\section{A.2. Proof of Theorem 1}

Under the null: $k_{1}=0$, when $\bar{r}=r$ we have

$\hat{F}_{t}=D F_{t}+o_{p}(1)$.

Let $D_{(i .)}$ denote the $i$ th row of $D$, and $D_{(\cdot j)}$ denote the $j$ th column of $D$. Define $\hat{\mathcal{F}}_{t}=D F_{t}$, and $\hat{\mathcal{F}}_{k t}=D_{(k \cdot)} \times F_{t}$ as the $k$ th element of $\hat{\mathcal{F}}_{t}$. Let $\hat{F}_{1 t}$ be the first element of $\hat{F}_{t}$, and $\hat{F}_{-1 t}=\left[\hat{F}_{2 t}, \ldots, \hat{F}_{r t}\right]^{\prime}$, while $\hat{\mathcal{F}}_{1 t}$ and $\hat{\mathcal{F}}_{-1 t}$ can be defined in the same way. Note that $\hat{\mathcal{F}}_{t}$ depends on $N$ and $T$. For simplicity, let $T \pi$ denote $[T \pi]$.

Note that under $H_{0}$, we allow for the existence of small breaks, so that the model can be written as $X_{i t}=\alpha_{i} F_{t}+e_{i t}+\eta_{i} G_{t}^{2}$. However, since $\eta_{i} G_{t}^{2}$ is $O_{p}(1 / \sqrt{N T})$ by Assumption 1 , we can use similar methods as in Appendix A.1 to show that an error term of this order can be ignored and that the asymptotic properties of $\hat{F}_{t}$ will not be affected (See Remark 5 of Bai, 2009). Therefore, for the sake of simplicity in the presentation below, we eliminate the last term and consider instead the model $X_{i t}=\alpha_{i} F_{t}+e_{i t}$ in the following Lemmas 9-13 required to prove Lemma 14 , which is the key result in the proof of Theorem 1.

\section{Lemma 9.}

$\sup _{\pi \in[0,1]}\left\|\frac{1}{T} \sum_{t=1}^{T \pi}\left(\hat{F}_{t}-\hat{\mathcal{F}}_{t}\right) F_{t}^{\prime}\right\|=O_{p}\left(\delta_{N, T}^{-2}\right)$.

Proof. The proof is similar to Lemma B.2 of Bai (2003). For details see our online appendix.

\section{Lemma 10.}

$\sup _{\pi \in[0,1]}\left\|\frac{1}{T} \sum_{t=1}^{T \pi} \hat{F}_{t} \hat{F}_{t}^{\prime}-\frac{1}{T} \sum_{t=1}^{T \pi} \hat{\mathcal{F}}_{t} \hat{\mathcal{F}}_{t}^{\prime}\right\|=O_{p}\left(\delta_{N, T}^{-2}\right)$.

Proof. Note that:

$$
\begin{aligned}
\frac{1}{T} \sum_{t=1}^{T \pi} \hat{F}_{t} \hat{F}_{t}^{\prime}-\frac{1}{T} \sum_{t=1}^{T \pi} \hat{\mathcal{F}}_{t} \hat{\mathcal{F}}_{t}^{\prime}= & \frac{1}{T} \sum_{t=1}^{T \pi} \hat{F}_{t} \hat{F}_{t}^{\prime}-\frac{1}{T} \sum_{t=1}^{T \pi}\left(D F_{t}\right)\left(F_{t}^{\prime} D^{\prime}\right) \\
= & \frac{1}{T} \sum_{t=1}^{T \pi} \hat{F}_{t}\left(\hat{F}_{t}^{\prime}-F_{t}^{\prime} D^{\prime}\right) \\
& +\frac{1}{T} \sum_{t=1}^{T \pi}\left(\hat{F}_{t}-D F_{t}\right)\left(F_{t}^{\prime} D^{\prime}\right) \\
= & \frac{1}{T} \sum_{t=1}^{T \pi}\left(\hat{F}_{t}-D F_{t}\right)\left(\hat{F}_{t}-D F_{t}\right)^{\prime} \\
& +\frac{1}{T} D \sum_{t=1}^{T \pi} F_{t}\left(\hat{F}_{t}-D F_{t}\right)^{\prime} \\
& +\frac{1}{T} \sum_{t=1}^{T \pi}\left(\hat{F}_{t}-D F_{t}\right)\left(F_{t}^{\prime} D^{\prime}\right) .
\end{aligned}
$$

Thus,

$$
\begin{aligned}
& \sup _{\pi \in[0,1]}\left\|\frac{1}{T} \sum_{t=1}^{T \pi} \hat{F}_{t} \hat{F}_{t}^{\prime}-\frac{1}{T} \sum_{t=1}^{T \pi} \hat{\mathcal{F}}_{t} \hat{\mathcal{F}}_{t}^{\prime}\right\| \\
& \leq \sup _{\pi \in[0,1] \|}\left\|\frac{1}{T} \sum_{t=1}^{T \pi}\left(\hat{F}_{t}-D F_{t}\right)\left(\hat{F}_{t}-D F_{t}\right)^{\prime}\right\|
\end{aligned}
$$

$$
\begin{aligned}
& +2\|D\| \sup _{\pi \in[0,1]}\left\|\frac{1}{T} \sum_{t=1}^{T \pi}\left(\hat{F}_{t}-D F_{t}\right) F_{t}^{\prime}\right\| \\
& \leq \frac{1}{T} \sum_{t=1}^{T}\left\|\hat{F}_{t}-D F_{t}\right\|^{2}+2\|D\| \sup _{\pi \in[0,1]}\left\|\frac{1}{T} \sum_{t=1}^{T \pi}\left(\hat{F}_{t}-D F_{t}\right) F_{t}^{\prime}\right\| .
\end{aligned}
$$

Since $\frac{1}{T} \sum_{t=1}^{T}\left\|\hat{F}_{t}-D F_{t}\right\|^{2}=O_{p}\left(\delta_{N, T}^{-2}\right)$ and $\sup _{\pi \in[0,1]} \| \frac{1}{T} \sum_{t=1}^{T \pi}\left(\hat{F}_{t}-\right.$ $\left.D F_{t}\right) F_{t}^{\prime} \|$ is $O_{p}\left(\delta_{N, T}^{-2}\right)$ by Lemma 9 , then the desired result is obtained.

The next two lemmas follow from Lemma 10 and Assumption 6:

\section{Lemma 11.}

$\sup _{\pi \in[0,1]}\left\|\frac{1}{\sqrt{T}} \sum_{t=1}^{T \pi} \hat{F}_{-1 t} \hat{F}_{1 t}-\frac{1}{\sqrt{T}} \sum_{t=1}^{T \pi} \hat{\mathcal{F}}_{-1 t} \hat{\mathcal{F}}_{1 t}\right\|=o_{p}(1)$.

Proof. See Lemma 10 and Assumption 6.

\section{Lemma 12.}

$\left\|\frac{1}{\sqrt{T}} \sum_{t=1}^{T} \hat{\mathcal{F}}_{-1 t} \hat{\mathcal{F}}_{1 t}^{\prime}\right\|=o_{p}(1)$.

Proof. By construction we have $\frac{1}{T} \sum_{t=1}^{T} \hat{F}_{-1 t} \hat{F}_{1 t}^{\prime}=0$, and then the result follows from Lemma 11.

Let $\Rightarrow$ denote weak convergence. $\mathcal{F}_{1 t}, \mathcal{F}_{-1 t}, D^{*}$ and $S$ are as defined in the paper (see Section 4.1). Then:

\section{Lemma 13.}

$\frac{1}{\sqrt{T}} \sum_{t=1}^{T \pi}\left(\mathcal{F}_{-1 t} \mathcal{F}_{1 t}-E\left(\mathcal{F}_{-1 t} \mathcal{F}_{1 t}\right)\right) \Rightarrow S^{1 / 2} W_{r-1}(\pi)$

for $\pi \in[0,1]$, where $W_{r-1}(\cdot)$ is a $r-1$ vector of independent Brownian motions on $[0,1]$.

Proof. The proof is a standard application of Functional CLT. For details see our online appendix.

\section{Lemma 14.}

$\frac{1}{\sqrt{T}} \sum_{t=1}^{T \pi} \hat{F}_{-1 t} \hat{F}_{1 t} \Rightarrow S^{1 / 2} \mathcal{B}_{r-1}^{0}(\pi)$

for $\pi \in[0,1]$, where the process $\mathcal{B}_{r-1}^{0}(\pi)=W_{r-1}(\pi)-\pi W_{r-1}(1)$ indexed by $\pi$ is a vector of Brownian Bridges on $[0,1]$.

Proof. If we show that

$\frac{1}{\sqrt{T}} \sum_{t=1}^{T \pi}\left[\mathcal{F}_{-1 t} \mathcal{F}_{1 t}-T^{-1} \sum_{s=1}^{T} \mathcal{F}_{-1 s} \mathcal{F}_{1 s}\right] \Rightarrow S^{1 / 2} \mathcal{B}_{r-1}^{0}(\pi)$

for $\pi \in[0,1]$ and

$$
\begin{aligned}
& \sup _{\pi \in[0,1]} \| \frac{1}{\sqrt{T}} \sum_{t=1}^{T \pi} \hat{\mathcal{F}}_{-1 t} \hat{\mathcal{F}}_{1 t} \\
& -\frac{1}{\sqrt{T}} \sum_{t=1}^{T \pi}\left[\mathcal{F}_{-1 t} \mathcal{F}_{1 t}-T^{-1} \sum_{s=1}^{T} \mathcal{F}_{-1 s} \mathcal{F}_{1 s}\right] \|=o_{p}(1),
\end{aligned}
$$

then the result follows from Lemma 11 . 
First note that

$$
\begin{aligned}
& \frac{1}{\sqrt{T}} \sum_{t=1}^{T \pi}\left[\mathcal{F}_{-1 t} \mathcal{F}_{1 t}-T^{-1} \sum_{s=1}^{T} \mathcal{F}_{-1 s} \mathcal{F}_{1 s}\right] \\
& =\frac{1}{\sqrt{T}} \sum_{t=1}^{T \pi}\left(\mathcal{F}_{-1 t} \mathcal{F}_{1 t}-E\left(\mathcal{F}_{-1 t} \mathcal{F}_{1 t}\right)\right) \\
& +\frac{1}{T} \sum_{t=1}^{T \pi}\left(\frac{1}{\sqrt{T}} \sum_{s=1}^{T}\left(\mathcal{F}_{-1 s} \mathcal{F}_{1 s}-E\left(\mathcal{F}_{-1 s} \mathcal{F}_{1 s}\right)\right)\right),
\end{aligned}
$$

hence (24) can be verified by applying Lemma 13.

To prove (25), we first define $D_{-1}$ as the second to last rows of $D$, and $D_{1}$ as the first row of $D . D_{-1}^{*}$ and $D_{1}^{*}$ are defined in the same manner. Then we have

$\hat{\mathcal{F}}_{-1 t} \hat{\mathcal{F}}_{1 t}=D_{-1} F_{t} F_{t}^{\prime} D_{1}^{\prime}$

and

$\mathcal{F}_{-1 t} \mathcal{F}_{1 t}=D_{-1}^{*} F_{t} F_{t}^{\prime} D_{1}^{*^{\prime}}$.

It follows that:

$$
\begin{aligned}
& \frac{1}{\sqrt{T}} \sum_{t=1}^{T \pi}\left(\hat{\mathcal{F}}_{-1 t} \hat{\mathcal{F}}_{1 t}-\mathcal{F}_{-1 t} \mathcal{F}_{1 t}\right) \\
& =\frac{1}{\sqrt{T}} \sum_{t=1}^{T \pi}\left(D_{-1} F_{t} F_{t}^{\prime} D_{1}^{\prime}-D_{-1} F_{t} F_{t}^{\prime} D_{1}^{*}+D_{-1} F_{t} F_{t}^{\prime} D_{1}^{*}-D_{-1}^{*} F_{t} F_{t}^{\prime} D_{1}^{*}\right) \\
& =D_{-1}\left(\frac{1}{\sqrt{T}} \sum_{t=1}^{T \pi} F_{t} F_{t}^{\prime}\right)\left(D_{1}^{\prime}-D_{1}^{*^{\prime}}\right) \\
& \quad+\left(D_{-1}-D_{-1}^{*}\right)\left(\frac{1}{\sqrt{T}} \sum_{t=1}^{T \pi} F_{t} F_{t}^{\prime}\right) D_{1}^{*^{\prime}} .
\end{aligned}
$$

Next, define $\overline{F F^{\prime}}=\frac{1}{T} \sum_{s=1}^{T} F_{s} F_{s}^{\prime}$, then:

$$
\begin{aligned}
\frac{1}{\sqrt{T}} \sum_{t=1}^{T \pi}\left(T^{-1} \sum_{s=1}^{s} \mathcal{F}_{-1 s} \mathcal{F}_{1 s}\right)=D_{-1}^{*}\left(\frac{1}{\sqrt{T}} \sum_{t=1}^{T \pi} \overline{F F^{\prime}}\right) D_{1}^{*^{\prime}} \\
=D_{-1}^{*}\left(\frac{1}{\sqrt{T}} \sum_{t=1}^{T \pi} \overline{F F^{\prime}}\right) D_{1}^{*^{\prime}}-D_{-1}\left(\frac{1}{\sqrt{T}} \sum_{t=1}^{T \pi} \overline{F F^{\prime}}\right) D_{1}^{*^{\prime}} \\
\quad+D_{-1}\left(\frac{1}{\sqrt{T}} \sum_{t=1}^{T \pi} \overline{F F^{\prime}}\right) D_{1}^{*^{\prime}} \\
\quad-D_{-1}\left(\frac{1}{\sqrt{T}} \sum_{t=1}^{T \pi} \overline{F F^{\prime}}\right) D_{1}^{\prime}+D_{-1}\left(\frac{1}{\sqrt{T}} \sum_{t=1}^{T \pi} \overline{F F^{\prime}}\right) D_{1}^{\prime} \\
=-\left(D_{-1}-D_{-1}^{*}\right)\left(\frac{1}{\sqrt{T}} \sum_{t=1}^{T \pi} \overline{F F^{\prime}}\right) D_{1}^{*^{\prime}} \\
\quad-D_{-1}\left(\frac{1}{\sqrt{T}} \sum_{t=1}^{T \pi} \overline{F F^{\prime}}\right)\left(D_{1}^{\prime}-D_{1}^{*^{\prime}}\right) \\
\quad+\frac{1}{\sqrt{T}} \sum_{t=1}^{T \pi}\left(\frac{1}{T} \sum_{s=1}^{T} \hat{\mathcal{F}}_{-1 s} \hat{\mathcal{F}}_{1 s}\right) .
\end{aligned}
$$

Combining the above results gives:

$$
\begin{aligned}
& \frac{1}{\sqrt{T}} \sum_{t=1}^{T \pi} \hat{\mathcal{F}}_{-1 t} \hat{\mathcal{F}}_{1 t}-\frac{1}{\sqrt{T}} \sum_{t=1}^{T \pi}\left[\mathcal{F}_{-1 t} \mathcal{F}_{1 t}-T^{-1} \sum_{s=1}^{T} \mathcal{F}_{-1 s} \mathcal{F}_{1 s}\right] \\
& =\frac{1}{\sqrt{T}} \sum_{t=1}^{T \pi}\left(\hat{\mathcal{F}}_{-1 t} \hat{\mathcal{F}}_{1 t}-\mathcal{F}_{-1 t} \mathcal{F}_{1 t}\right)+\frac{1}{\sqrt{T}} \sum_{t=1}^{T \pi}\left(T^{-1} \sum_{s=1}^{T} \mathcal{F}_{-1 s} \mathcal{F}_{1 s}\right)
\end{aligned}
$$

$$
\begin{aligned}
= & D_{-1}\left(\frac{1}{\sqrt{T}} \sum_{t=1}^{T \pi}\left(F_{t} F_{t}^{\prime}-\overline{F F^{\prime}}\right)\right)\left(D_{1}^{\prime}-D_{1}^{*^{\prime}}\right) \\
& +\left(D_{-1}-D_{-1}^{*}\right)\left(\frac{1}{\sqrt{T}} \sum_{t=1}^{T \pi}\left(F_{t} F_{t}^{\prime}-\overline{F F^{\prime}}\right)\right) D_{1}^{*^{\prime}} \\
& +\frac{1}{\sqrt{T}} \sum_{t=1}^{T \pi}\left(\frac{1}{T} \sum_{s=1}^{T} \hat{\mathcal{F}}_{-1 s} \hat{\mathcal{F}}_{1 s}\right) .
\end{aligned}
$$

Following similar arguments as in Lemma 13, it can proved that

$\sup _{\pi \in[0,1]}\left\|\frac{1}{\sqrt{T}} \sum_{t=1}^{T \pi}\left(F_{t} F_{t}^{\prime}-\overline{F F^{\prime}}\right)\right\|=O_{p}(1)$.

Moreover, it is easy to check that $\|D\|=O_{p}(1)$ and $\left\|D-D^{*}\right\|$ $=o_{p}(1)$ (See Bai, 2003). Finally, $\left\|\frac{1}{\sqrt{T}} \sum_{s=1}^{T} \hat{\mathcal{F}}_{-1 s} \hat{\mathcal{F}}_{1 s}\right\|$ is $o_{p}(1)$ by Lemma 12 . Then (25) holds and we obtain the desired conclusion.

\section{Theorem 1.}

Proof. The results for LM and Sup-LM tests follow from Assumption 9, Lemma 14, and Continuous Mapping Theorem.

For the Wald and Sup-Wald tests, notice that:

$$
\begin{aligned}
\sqrt{T} & \left(\hat{c}_{1}(\pi)-\hat{c}_{2}(\pi)\right) \\
= & \left(1 / T \sum_{t=1}^{\tau} \hat{F}_{-1 t} \hat{F}_{-1 t}^{\prime}\right)^{-1}\left(1 / \sqrt{T} \sum_{t=1}^{\tau} \hat{F}_{-1 t} \hat{F}_{1 t}\right) \\
& -\left(1 / T \sum_{t=\tau+1}^{T} \hat{F}_{-1 t} \hat{F}_{-1 t}^{\prime}\right)^{-1}\left(1 / \sqrt{T} \sum_{t=\tau+1}^{T} \hat{F}_{-1 t} \hat{F}_{1 t}\right) \\
= & {\left[\left(1 / T \sum_{t=1}^{\tau} \hat{F}_{-1 t} \hat{F}_{-1 t}^{\prime}\right)^{-1}+\left(I-1 / T \sum_{t=1}^{\tau} \hat{F}_{-1 t} \hat{F}_{-1 t}^{\prime}\right)^{-1}\right] } \\
& \times\left(1 / \sqrt{T} \sum_{t=1}^{\tau} \hat{F}_{-1 t} \hat{F}_{1 t}\right) .
\end{aligned}
$$

By Lemma 10 and that $D-D^{*}=o_{p}(1)$, we have:

$$
\begin{aligned}
1 / T \sum_{t=1}^{\tau} \hat{F}_{-1 t} \hat{F}_{-1 t}^{\prime} & =\pi \frac{1}{\tau} \sum_{t=1}^{\tau} \hat{F}_{-1 t} \hat{F}_{-1 t}^{\prime} \\
& =\pi \frac{1}{\tau} \sum_{t=1}^{\tau} \mathcal{F}_{-1 t} \mathcal{F}_{-1 t}^{\prime}+o_{p}(1) .
\end{aligned}
$$

When $\tau=T(\pi=\tau / T=1)$, this implies

$$
I_{r-1}=1 / T \sum_{t=1}^{T} \hat{F}_{-1 t} \hat{F}_{-1 t}^{\prime}=\frac{1}{T} \sum_{t=1}^{T} \mathcal{F}_{-1 t} \mathcal{F}_{-1 t}^{\prime}+o_{p}(1) .
$$

Notice that $E\left(\mathcal{F}_{-1 t} \mathcal{F}_{-1 t}^{\prime}\right)=I_{r-1}$, because $E\left(\mathcal{F}_{t} \mathcal{F}_{t}^{\prime}\right)=D^{*} \Sigma_{F} D^{*}=$ $V^{-1 / 2} \Gamma^{\prime} \Sigma_{\Lambda}^{1 / 2} \Sigma_{F} \Sigma_{\Lambda}^{1 / 2} \Gamma V^{-1 / 2}=I_{r}$. Applying law of large numbers to (26) gives:

$1 / T \sum_{t=1}^{\tau} \hat{F}_{-1 t} \hat{F}_{-1 t}^{\prime} \stackrel{p}{\rightarrow} \pi I_{r-1}$

as $N$ and $T$ go to infinity. Then it follows from Lemma 14 that:

$\sqrt{T}\left(\hat{c}_{1}(\pi)-\hat{c}_{2}(\pi)\right) \Rightarrow \frac{S^{1 / 2} \mathscr{B}_{r-1}^{0}(\pi)}{\pi(1-\pi)}$

and the limit distributions of the Wald and Sup-Wald tests follow easily. 


\section{A.3. Consistent estimator of $S$}

We now discuss the consistent estimator of $S$ using the HAC estimator of Newey and West (1987). Recall that $S=$ $\lim \operatorname{Var}\left(\frac{1}{\sqrt{T}} \sum_{t=1}^{T} \mathcal{F}_{-1 t} \mathcal{F}_{1 t}\right)$. Notice that $E\left(\mathcal{F}_{-1 t} \mathcal{F}_{1 t}\right)=0$ as shown above.

First, we define the infeasible estimator of $S$ :

$\hat{S}(\mathcal{F})=\hat{\Gamma}_{0}(\mathcal{F})+\sum_{j=1}^{m} w(j, m)\left[\hat{\Gamma}_{j}(\mathcal{F})+\hat{\Gamma}_{j}(\mathcal{F})^{\prime}\right]$

where $m=o\left(T^{\frac{1}{4}}\right), w(j, m)=1-\frac{j}{m+1}$ is the Bartlett kernel, and $\hat{\Gamma}_{j}(\mathcal{F})=\frac{1}{T} \sum_{t=j+1}^{T} \mathcal{F}_{-1 t} \mathcal{F}_{1 t} \mathcal{F}_{1 t-j} \mathcal{F}_{-1 t-j}^{\prime}$.

Since the above estimator is a HAC estimator, it is natural to make the following assumption:

Assumption 11. $\|\hat{S}(\mathcal{F})-S\|=o_{p}(1)$.

Next we consider a feasible estimator of $S$ where $\mathcal{F}_{t}$ is replaced by $\hat{F}_{t}$ :

$\hat{S}(\hat{F})=\hat{\Gamma}_{0}(\hat{F})+\sum_{j=1}^{m} w(j, m)\left[\hat{\Gamma}_{j}(\hat{F})+\hat{\Gamma}_{j}(\hat{F})^{\prime}\right]$

where

$\hat{\Gamma}_{j}(\hat{F})=\frac{1}{T} \sum_{t=j+1}^{T} \hat{F}_{-1 t} \hat{F}_{1 t} \hat{F}_{1 t-j} \hat{F}_{-1 t-j}^{\prime}$

then we have the following results:

Proposition 3. Assume that Assumptions 1-11 hold, under the null $H_{0}: k_{1}=0$, we have

$\|\hat{S}(\hat{F})-S\|=o_{p}(1)$.

Proof. Given Assumption 11, it suffices to show that

$\|\hat{S}(\hat{F})-\hat{S}(\mathcal{F})\|=o_{p}(1)$.

It is easy to see that:

$\|\hat{S}(\hat{F})-\hat{S}(\mathcal{F})\| \leq 2 \sum_{j=0}^{m}\left\|\hat{\Gamma}_{j}(\hat{F})-\hat{\Gamma}_{j}(\mathcal{F})\right\|$.

For the right-hand side we obtain that:

$$
\begin{aligned}
\sup _{0 \leq j \leq m} & \left\|\hat{\Gamma}_{j}(\hat{F})-\hat{\Gamma}_{j}(\mathcal{F})\right\| \\
\leq & \sup _{0 \leq j \leq m} \frac{1}{T} \sum_{t=j+1}^{T}\left\|\hat{F}_{-1 t} \hat{F}_{1 t} \hat{F}_{1 t-j} \hat{F}_{-1 t-j}^{\prime}-\mathcal{F}_{-1 t} \mathcal{F}_{1 t} \mathcal{F}_{1 t-j} \mathcal{F}_{-1 t-j}^{\prime}\right\| \\
\leq & \sup _{0 \leq j \leq m} \frac{1}{T} \sum_{t=j+1}^{T}\left\|\left(\hat{F}_{1 t} \hat{F}_{-1 t}-\mathcal{F}_{1 t} \mathcal{F}_{-1 t}\right) \hat{F}_{1 t-j} \hat{F}_{-1 t-j}^{\prime}\right\| \\
& +\sup _{0 \leq j \leq m} \frac{1}{T} \sum_{t=j+1}^{T}\left\|\mathcal{F}_{1 t} \mathcal{F}_{-1 t}\left(\hat{F}_{1 t-j} \hat{F}_{-1 t-j}^{\prime}-\mathcal{F}_{1 t-j} \mathcal{F}_{-1 t-j}^{\prime}\right)\right\| \\
\leq & \sqrt{\frac{1}{T} \sum_{t=1}^{T}\left\|\hat{F}_{1 t} \hat{F}_{-1 t}-\mathcal{F}_{1 t} \mathcal{F}_{-1 t}\right\|^{2}} \\
& \times\left(\sqrt{\frac{1}{T} \sum_{t=1}^{T}\left\|\hat{F}_{1 t} \hat{F}_{-1 t}\right\|^{2}}+\sqrt{\frac{1}{T} \sum_{t=1}^{T}\left\|\mathcal{F}_{1 t} \mathcal{F}_{-1 t}\right\|^{2}}\right)
\end{aligned}
$$

$\frac{1}{T} \sum_{t=1}^{T}\left\|\hat{F}_{1 t} \hat{F}_{-1 t}\right\|^{2} \leq \frac{1}{T} \sum_{t=1}^{T}\left\|\hat{F}_{t}\right\|^{4}=O_{p}(1)$ by Lemma 5 of $\mathrm{HI}$ (2012), and $\frac{1}{T} \sum_{t=1}^{T}\left\|\mathcal{F}_{1 t} \mathcal{F}_{-1 t}\right\|^{2} \leq \frac{1}{T} \sum_{t=1}^{T}\left\|\mathscr{F}_{t}\right\|^{4}=O_{p}(1)$ by Assumption 2. Furthermore,

$\frac{1}{T} \sum_{t=1}^{T}\left\|\hat{F}_{1 t} \hat{F}_{-1 t}-\mathcal{F}_{1 t} \mathcal{F}_{-1 t}\right\|^{2}$

$\leq \frac{1}{T} \sum_{t=1}^{T}\left\|\left(\hat{F}_{1 t}-\mathcal{F}_{1 t}\right) \hat{F}_{-1 t}\right\|^{2}+\frac{1}{T} \sum_{t=1}^{T}\left\|\left(\hat{F}_{-1 t}-\mathcal{F}_{-1 t}\right) \mathcal{F}_{1 t}\right\|^{2}$

$\leq \sqrt{\frac{1}{T} \sum_{t=1}^{T}\left\|\hat{F}_{t}-\mathcal{F}_{t}\right\|^{4}}\left(\sqrt{\frac{1}{T} \sum_{t=1}^{T}\left\|\hat{F}_{t}\right\|^{4}}+\sqrt{\frac{1}{T} \sum_{t=1}^{T}\left\|\mathcal{F}_{t}\right\|^{4}}\right)$.

It can be proved that $\frac{1}{T} \sum_{t=1}^{T}\left\|\hat{F}_{t}-\mathcal{F}_{t}\right\|^{4}=O_{p}(1 / T)+O_{p}\left(1 / N^{2}\right)$ (very similar to Theorem 2 of HI, 2012). Then, under the assumption that $\sqrt{T} / N \rightarrow 0$, it follows that

$$
\begin{aligned}
& \sup _{0 \leq j \leq m} \frac{1}{T} \sum_{t=j+1}^{T}\left\|\hat{F}_{1 t} \hat{F}_{-1 t} \hat{F}_{1 t-j} \hat{F}_{-1 t-j}^{\prime}-\mathcal{F}_{1 t} \mathcal{F}_{-1 t} \mathcal{F}_{1 t-j} \mathcal{F}_{-1 t-j}^{\prime}\right\| \\
& \quad=O_{p}\left(T^{-1 / 4}\right),
\end{aligned}
$$

which implies $\|\hat{S}(\hat{F})-\hat{S}(\mathcal{F})\|=o_{p}(1)$ given that $m=o\left(T^{1 / 4}\right)$.

\section{References}

Ahn, S.C., Horenstein, A.R., 2013. Eigenvalue ratio test for the number of factors. Econometrica 81 (3), 1203-1227.

Andrews, D., 1993. Tests for parameter instability and structural change with unknown change point. Econometrica 61 (4), 821-856.

Bai, J., 2003. Inferential theory for factor models of large dimensions. Econometrica $71(1), 135-171$.

Bai, J., 2009. Panel data models with interactive fixed effects. Econometrica 77 (4), 1229-1279.

Bai, J., Ng, S., 2002. Determining the number of factors in approximate factor models. Econometrica 70 (1), 191-221.

Bai, J., Ng, S., 2006a. Confidence intervals for diffusion index forecasts and inference for factor-augmented regressions. Econometrica 74 (4), 1133-1150.

Bai, J., Ng. S., 2006b. Evaluating latent and observed factors in macroeconomics and finance. J. Econometrics 131 (1-2), 507-537.

Bai, J., Perron, P., 1998. Estimating and testing linear models with multiple structural changes. Econometrica 66 (1), 47-78.

Bai, J., Perron, P., 2003. Computation and analysis of multiple structural change models. J. Appl. Econometrics 18 (1), 1-22.

Banerjee, A., Marcellino, M., Masten, I., 2008. Forecasting macroeconomic variables using diffusion indexes in short samples with structural change. CEPR. DP. 6706.

Bates, B., Plagborg-Moller, M. Stock, J., Watson, M., 2013. Consistent factor estimation in dynamic factor models with structural instability. J. Econometrics 177 (2), 289-304

Bernanke, B., Boivin, J., Eliasz, P., 2005. Measuring the effects of monetary policy: a factor-augmented vector autoregressive (FAVAR) approach. Quart. J. Econom. 120 (1), 387-422.

Breitung, J., Eickmeier, S., 2011. Testing for structural breaks in dynamic factor models. J. Econometrics 163 (1), 71-84

Brown, R., Durbin, J., Evans, J., 1975. Techniques for testing the constancy of reoression relationships over time. J. R. Stat. Soc. Ser B Stat Methodol 149-192.

Charnavoki, V., Dolado, J., 2012. The effects of global shocks on small commodityexporting economies: new evidence from Canada. CEPR. DP. 8825.

Chen, B., Hong, Y., 2012. Testing for smooth structural changes in time series models via nonparametric regression. Econometrica 80 (3), 1157-1183.

Chen, L., 2012, Identifying observed factors in high dimensional factor models. Universidad Carlos III de Madrid, Working Paper.

Chen, N., Roll, R., Ross, S., 1986. Economic forces and the stock market. J. Business 383-403.

Diebold, F., Chen, C., 1996. Testing structural stability with endogenous breakpoint a size comparison of analytic and bootstrap procedures. J. Econometrics 70 (1), 221-241.

Fernández-Villaverde, J., Guerrón-Quintana, P.A., Rubio-Ramírez, J., 2010. Reading the recent monetary history of the US, 1959-2007. Federal Reserve Board of St. Louis Review, 92, pp. 1-28.

Forni, M., Lippi, M., 2001. The generalized dynamic factor model: representation theory. Econometric Theory 17 (06), 1113-1141.

Gagliardini, P., Gouriéroux, C., 2011. Efficiency in large dynamic panel models with common factor. U. of Toronto (mimeo).

Gali, J., Gambetti, L., 2009. On the sources of the great moderation. Amer. Econom. J. Macroeconom. 1 (1), 26-57. 
Giannone, D., 2007. Discussion on: "forecasting in dynamic factor models subject to structural instability" by Stock and Watson. In: New Developments in Dynamic Factor Modelling. Bank of England, Centre for Central Banking Studies.

Han, X., Inoue, A., 2012. Tests for parameter instability in dynamic factor models. North Carolina State University, Working Paper.

Hansen, B., 2000. Testing for structural change in conditional models. J. Econometrics 97 (1), 93-115.

Kim, D., Perron, P., 2009. Unit root tests allowing for a break in the trend function at an unknown time under both the null and alternative hypotheses. J. Econometrics 148 (1), 1-13.

Newey, W., West, K., 1987. A simple, positive semi-definite, heteroskedasticity and autocorrelation consistent covariance matrix. Econometrica 55 (3), 703-708.

Onatski, A., 2009. Testing hypotheses about the number of factors in large factor models. Econometrica 77 (5), 1447-1479.
Onatski, A., 2010. Determining the number of factors from empirical distribution of eigenvalues. Rev. Econom. Stat. 92 (4), 1004-1016.

Perron, P., 2006. Dealing with structural breaks. In: Palgrave handbook of econometrics, vol. 1. pp. 278-352.

Rudebusch, G., Wu, T., 2008. A macro-finance model of the term structure, monetary policy and the economy. The Economic Journal 118 (530), 906-926.

Shanken, J., Weinstein, M., 2006. Economic forces and the stock market revisited. J. Empirical Fin. 13 (2), 129-144.

Stock, J., Watson, M., 2002. Forecasting using principal components from a large number of predictors. J. Amer. Statist. Assoc. 97 (460), 1167-1179.

Stock, J., Watson, M., 2009. Forecasting in dynamic factor models subject to structural instability. In: The Methodology and Practice of Econometrics. A Festschrift in Honour of David F. Hendry. pp. 173-205. 